\title{
Diversidad y distribución de la familia Asteraceae en México
}

\author{
José LUIS VILLASEÑor
}

Botanical Sciences

96 (2): 332-358, 2018

DOI: $10.17129 /$ botsci.1872

Received:

October 2nd, 2017

Accepted:

February 18th, 2018

Associated Editor:

Guillermo Ibarra-Manríquez
Instituto de Biología,

Universidad Nacional

Autónoma de México. Ciudad de México. México

vrios@ib.unam.mx

This is an open access article distributed under the terms of the Creative Commons Attribution License CC BY-NC (4.0) international.

\section{Resumen}

Antecedentes: La familia Asteraceae (o Compositae) en México ha llamado la atención de prominentes botánicos en las últimas décadas, por lo que cuenta con una larga tradición de investigación de su riqueza florística. Se cuenta, por lo tanto, con un gran acervo bibliográfico que permite hacer una síntesis y actualización de su conocimiento florístico a nivel nacional.

Pregunta: ¿Cuál es la riqueza actualmente conocida de Asteraceae en México? ¿Cómo se distribuye a lo largo del territorio nacional? ¿Qué géneros o regiones requieren de estudios más detallados para mejorar el conocimiento de la familia en el país?

Área de estudio: México.

Métodos: Se llevó a cabo una exhaustiva revisión de literatura florística y taxonómica, así como la revisión de unos 200,000 ejemplares de herbario, depositados en más de 20 herbarios, tanto nacionales como del extranjero.

Resultados: México registra 26 tribus, 417 géneros y 3,113 especies de Asteraceae, de las cuales 3,050 son especies nativas y 1,988 (63.9\%) son endémicas del territorio nacional. Los géneros más relevantes, tanto por el número de especies como por su componente endémico, son Ageratina (164 y 135, respectivamente), Verbesina $(164,138)$ y Stevia $(116,95)$. Los estados con mayor número de especies son Oaxaca (1,040), Jalisco (956), Durango (909), Guerrero (855) y Michoacán (837). Los biomas con la mayor riqueza de géneros y especies son el bosque templado $(1,906)$ y el matorral xerófilo $(1,254)$. Solamente 31 géneros no cuentan con revisión taxonómica o con la suficiente información para llevar a cabo la correcta determinación de sus especies, aunque para muchos otros es necesario actualizar la información taxonómica por las nuevas especies descritas o los cambios taxonómicos de sus especies. El esfuerzo de recolecta a lo largo del país revela que existen todavía importantes regiones que requieren exploración para documentar mejor la riqueza de especies de Asteraceae.

Conclusiones: La evaluación de la literatura y la consulta de especímenes herborizados muestran un escenario optimista en cuanto al conocimiento florístico-taxonómico de la familia en México. Sin embargo, todavía se tienen amplias zonas del territorio (en especial su porción norte) que no han sido debidamente exploradas. Sin duda, una mayor exploración de tales zonas modificará las cifras discutidas, cuando se conozca mejor su riqueza de Asteraceae.

Palabras clave: Compositae, endemismo, diversidad alfa, riqueza florística.

\section{Abstract}

Background: In Mexico, the family Asteraceae (or Compositae) has attracted the attention of prominent botanists in the last decades; for this reason, it has a long tradition of investigation of its floristic richness. The family has a large bibliographic collection that allows to synthesize and update its floristic knowledge.

Question: Which is the current richness of Asteraceae in Mexico? How Asteraceae is distributed throughout this country? Which genera or regions require more detailed studies to improve our knowledge of the family in the country?

Study site: Mexico.

Methods: An exhaustive review of floristic and taxonomic literature was carried out, as well as the revision of about 200,000 herbarium specimens deposited in more than 20 herbaria both nationally and abroad.

Results: Mexico currently records 26 tribes, 417 genera and 3,113 species of Asteraceae, of which 3,050 are native species and $1,988(63.9 \%)$ endemic to this country. The most important genera, 
both for the number of species and for their endemic component, are Ageratina (164 and 135, respectively), Verbesina $(164,138)$ and Stevia $(116,95)$. The states with the greatest number of species are Oaxaca (1,040), Jalisco (956), Durango (909), Guerrero (855) and Michoacán (837), while the biomes with the greatest richness are the temperate forest $(1,906)$ and the xerophytic scrub $(1,254)$. Only 31 genera do not have taxonomic revision or enough information to carry out the correct determination of their species, although for many others it is necessary to update the taxonomic information by the new discoveries or the taxonomic changes at species level. The collection effort throughout the country reveals that there are still important regions that require exploration to better document their species richness.

Conclusions: The evaluation of the literature and the review of herbarium specimens shows an optimistic scenario regarding the knowledge of the family in Mexico. However, there are still large areas of the territory that once properly explored, will modify the figures here discussed about their Asteraceae richness.

Key Words: Alpha diversity, Compositae, endemism, floristic richness.

a familia Asteraceae o Compositae es la más grande de la flora de México por su número de géneros y especies (Villaseñor 2016). El género tipo de la familia es Aster, publicado por Linneo en su magna obra Species plantarum $(1,753)$, que constituye la base de la nomenclatura contemporánea. El nombre alternativo de Compositae (nomen conservandum) antecede la propuesta Linneana y hace referencia a la conspicua disposición de la inflorescencia básica en la familia, un capítulo o cabezuela que semeja una flor ("pseudantio"), pero que en realidad son pocas a muchas flores sésiles e insertas en una estructura parecida a un cáliz denominado involucro (estructura formada por un conjunto de brácteas involucrales). Muy pocas familias tienen dispuestas sus flores con este arreglo, por ejemplo, Calyceraceae o algunas especies de Goodeniaceae, por lo que Asteraceae, desde los tiempos de Teofrasto (alrededor de 320 A.C.), ha sido reconocida como un grupo natural (Small 1917). En la actualidad, otros rasgos, tanto morfológicos como derivados de estudios de biología molecular, han sido añadidos como características que confirman su monofilia; uno de ellos es la conformación peculiar de su androceo, con los filamentos libres y las anteras fusionadas por un tejido (estambres singenesios o sinantéreos). Por esta razón, muchos consideran a la "Sinanterología" como el estudio de esta interesante familia. Otro rasgo peculiar en la familia es su cáliz modificado en una estructura muy variable denominada vilano.

La familia se distribuye a lo largo de todo México, desde las dunas o vegetación costera hasta los picos nevados de las altas montañas. Diversos autores han tratado de explicar por qué la amplia distribución geográfica de la familia y su gran riqueza de especies; entre las respuestas se cuentan la eficiente capacidad de dispersión proporcionada por el vilano o su gran plasticidad genética. Esta última se expresa en una amplia diversidad de metabolitos secundarios que sintetizan como estrategias de protección contra depredadores o competidores o en la gran variabilidad fenotípica (formas de crecimiento o estrategias de reproducción mostradas por la variabilidad morfológica y sexual de las cabezuelas y las flores) y genotípica, que permite a muchas especies adaptarse a diversas condiciones ecológicas, muchas veces como malezas (Leppik 1970, Carlquist 1976, Cronquist 1981, Cabrera-Rodríguez \& Villaseñor 1987).

Seguramente la diversidad de formas de crecimiento, variabilidad morfológica, amplia distribución geográfica, pero sobre todo su diversidad taxonómica, provoca bloqueos psicológicos en muchas personas (incluyendo taxónomos o botánicos) que intentan identificar a sus miembros. Sin embargo, conforme uno va conociendo la familia, poco a poco se da cuenta de su simplicidad y reconoce que la familia no es tan compleja como otros grupos o familias de plantas. George Bentham (1873) ya comentaba desde hace siglo y medio que la familia "es la más uniforme y por lo tanto el más natural de todos los órdenes de fanerógamas ... y no puedo recordar una sola de sus especies que sin duda pueda ser ubicada en el orden". Otra evidencia más actual de ello es que la familia es de las pocas en la flora de México que desde hace casi cuatro décadas cuenta con claves para la identificación de sus géneros (Rzedowski 1978, Murguía \& Villaseñor 1996), lo que ha facilitado grandemente el trabajo rutinario de determinación a nivel genérico. Sin embargo, es indudable que tratar de identificar a especie la gran riqueza de la familia, es en ocasiones difícil, especialmente cuando no se tienen suficientes y adecuadas claves de identificación a nivel regional o local. 
El conocimiento que se cuenta ahora sobre la familia descansa en los antecedentes que legaron pioneros del siglo XIX, como Augustin P. de Candolle (1778-1841), Henri Cassini (17811832), George Bentham (1800-1884), Christian F. Lessing (1809-1862), Asa Gray (1810-1888), Sidney F. Blake (1814-1888), Benjamín L. Robinson (1864-1935) o Jesse M. Greenman (18671951). Otros botánicos destacados del siglo XX igualmente han contribuido de manera importante a generar la estructura del conocimiento sistemático de la familia; entre ellos se pueden mencionar a Arthur Cronquist (1919-1992), Sherwin Carlquist (1930-), Robert M. King (19302007), Harold E. Robinson (1932-) o Kare Bremer (1948-). Varios botánicos, tanto nacionales como extranjeros han contribuido de manera importante a generar el amplio conocimiento florístico-taxonómico que se tiene de la familia en México; destacan de manera especial los doctores Rogers McVaugh (1909-2009) y Billie L. Turner (1925-). Sin embargo, el principal generador de información para la familia y motivador de varias generaciones de botánicos que ha tenido el país es el doctor Jerzy Rzedowski (1926-); sus trabajos son clásicos y de consulta obligada aún fuera de México.

Una revisión de la literatura para documentar qué botánicos mexicanos se han involucrado en este último siglo en aspectos florístico-taxonómicos de las Asteraceae no revela la existencia de más de 50. Su aportación va dirigida principalmente a la descripción de especies o géneros y a la publicación de tratamientos florísticos de la familia, pocas veces a la publicación de revisiones taxonómicas o análisis filogenéticos. Es importante subrayar además que casi todos ellos se concentran en no más de siete instituciones nacionales. Destacan el Instituto de Ecología, A. C. (Pátzcuaro, Michoacán), donde los doctores Jerzy Rzedowski y Graciela Calderón de Rzedowski han dedicado mucho esfuerzo en conocer la familia, especialmente en la región del Bajío. Otros grupos lo constituyen José Luis Villaseñor, sus estudiantes y colaboradores del Instituto de Biología, UNAM; el cuerpo académico del Centro Universitario de Ciencias Biológicas y Agropecuarias, de la Universidad de Guadalajara, encabezado por el doctor Aarón Rodríguez, la M. en C. Mollie Harker y sus estudiantes de posgrado; el grupo coordinado por la doctora Socorro González-Elizondo, del Centro Interdisciplinario de Investigación para el Desarrollo Integral Regional, Unidad Durango, del IPN; el grupo encabezado por el doctor José Ángel Villarreal, de la Universidad Autónoma Agraria Antonio Narro, en Saltillo, Coahuila, así como el coordinado por el doctor José Luis León de la Luz, del Centro de Investigaciones Biológicas del Noroeste en La Paz, Baja California Sur.

Conocimiento filogenético de la familia Asteraceae. Como ya se indicó, desde hace más de dos siglos se consideraba a las Compuestas como un grupo natural. Sin embargo, con las ideas y enfoque novedoso sobre relaciones filogenéticas propuesto por Willi Henning (1913-1976), se fundamentó la monofilia de la familia con base en características morfológicas y se comenzaron a evaluar las relaciones filogenéticas entre sus miembros (e.g., Carlquist 1976, Heywood et al. 1977, Robinson 1981, Bremer 1987, 1994, King \& Robinson 1987). Con la implementación en la sistemática de los estudios moleculares de ADN, Jansen \& Palmer (1987) confirmaron bajo este enfoque la monofilia de la familia y sus tribus como son actualmente circunscritas; desde entonces una plétora de publicaciones sobre el tema ha sido publicada. Un recuento reciente de todos esos resultados, combinándolos con la evidencia morfológica ha sido resumida por Funk et al. (2009) para toda la familia; a manera de ejemplos más específicos se pueden citar los trabajos a nivel de tribus: Heliantheae (Schilling \& Panero 2002, 2011), Tageteae (Loockerman et al. 2003), Vernonieae (Keeley et al. 2007), Senecioneae (Pelser et al. 2007) o Liabeae (Funk et al. 2012).

Bajo esta perspectiva filogenética, la familia actualmente está dividida en 45 tribus (Funk et al. 2009), de las cuales 24 contienen especies nativas en México (Tabla 1); otras dos tribus (Arctotideae y Calenduleae) registran especies introducidas (exóticas), al parecer ya naturalizadas o en proceso de naturalización. En total, Asteraceae cuenta con 417 géneros y 3,113 especies (Tabla 1), cifras que lo ubican entre los países con la mayor riqueza en miembros de esta familia, ya que supera de manera importante las cifras reportadas para otros países neotropicales con gran riqueza florística o mayor superficie como, por ejemplo, Argentina (1,307), Brasil (1,966), Colombia (1,420), Ecuador (918) o Perú (1,432) (Brako \& Zarucchi 1993, Katinas et al. 2007, BFG 2015, Rangel-Churio 2015). 
Tabla 1. Tribus de la familia Asteraceae con especies tanto nativas como introducidas (exóticas) en México. l= Tribu solamente con elementos exóticos en la flora de México.

\begin{tabular}{|c|c|c|c|c|c|c|}
\hline \multirow[b]{2}{*}{ Tribu } & \multicolumn{3}{|c|}{ Géneros } & \multicolumn{3}{|c|}{ Especies } \\
\hline & Total & Endémicos & Exóticos & Total & Endémicas & Exóticas \\
\hline Anthemideae & 11 & - & 8 & 30 & 3 & 16 \\
\hline Arctotideae (I) & 2 & - & 2 & 2 & - & 2 \\
\hline Astereae & 52 & 5 & 1 & 380 & 200 & 2 \\
\hline Bahieae & 14 & 1 & - & 48 & 23 & - \\
\hline Calenduleae (I) & 1 & - & 1 & 1 & - & 1 \\
\hline Cardueae & 8 & - & 6 & 57 & 28 & 11 \\
\hline Chaenactideae & 1 & - & - & 8 & 1 & - \\
\hline Cichorieae & 29 & 1 & 10 & 85 & 19 & 17 \\
\hline Coreopsideae & 14 & 2 & - & 199 & 144 & - \\
\hline Eupatorieae & 46 & 6 & - & 619 & 433 & - \\
\hline Gnaphalieae & 15 & 1 & 1 & 75 & 25 & 4 \\
\hline Gochnatieae & 1 & - & - & 7 & 6 & - \\
\hline Helenieae & 12 & 1 & - & 44 & 14 & - \\
\hline Heliantheae & 77 & 13 & 1 & 650 & 464 & 1 \\
\hline Inuleae & 4 & - & - & 12 & 2 & - \\
\hline Liabeae & 5 & 1 & - & 25 & 15 & - \\
\hline Madieae & 14 & 2 & - & 35 & 7 & - \\
\hline Millerieae & 26 & 5 & - & 174 & 131 & - \\
\hline Mutisieae & 3 & - & - & 13 & 7 & - \\
\hline Nassauvieae & 3 & - & - & 97 & 83 & - \\
\hline Neurolaeneae & 5 & 1 & - & 28 & 19 & - \\
\hline Onoserideae & 1 & - & - & 1 & - & - \\
\hline Perityleae & 5 & 2 & - & 51 & 40 & - \\
\hline Senecioneae & 22 & 3 & 3 & 225 & 165 & 6 \\
\hline Tageteae & 27 & 10 & - & 173 & 112 & 1 \\
\hline Vernonieae & 19 & 3 & 2 & 74 & 47 & 2 \\
\hline Total & 417 & 57 & 35 & 3,113 & 1,988 & 63 \\
\hline
\end{tabular}

Evaluación del conocimiento y nivel de exploración de la familia Asteraceae en México. Como se indicó anteriormente, fue Jerzy Rzedowski quién cimentó y sintetizó las bases del conocimiento que se tiene de la familia en México. Sus trabajos clásicos sobre fitogeografía (Rzedowski 1972) y sobre identificación botánica (Rzedowski 1978) pueden considerarse pioneros en el estudio de la familia a nivel nacional. Posteriormente, Cabrera-Rodríguez \& Villaseñor (1987) presentaron una primera evaluación a nivel genérico sobre el estado del conocimiento florístico-taxonómico de la familia en México. Entre sus principales conclusiones se destacaba el importante nivel de conocimiento que se tenía para ese tiempo de la familia, identificando regiones potenciales para exploración y así enriquecer mejor dicho conocimiento. Tiempo después, Villaseñor (1993) presenta una primera síntesis de la riqueza a nivel nacional, reportando el número de especies registradas hasta la fecha en los diferentes estados en que se divide el país. De manera particular, Villaseñor (1990) analizó la riqueza del elemento endémico de Asteraceae a nivel de género, discutiendo patrones biogeográficos bastante coincidentes a lo reportado por Rzedowski (1991) para toda la flora nacional, definiendo los límites de distribución de dichos géneros más allá de las fronteras políticas, lo que actualmente conocemos como MegaMéxico I y II (Rzedowski 1991). Finalmente, Villaseñor et al. $(1998,2005,2007)$ discutieron el papel de la familia en posibles estrategias de conservación y de estimación de la diversidad a nivel nacional, en tanto que 
Balleza \& Villaseñor (2011), Suárez-Mota \& Villaseñor (2011) y Suárez-Mota et al. (2015a, 2017), lo hicieron a nivel regional.

Entre los estudios taxonómicos o sistemáticos enfocados a la flora de México y publicados en los últimos años se pueden mencionar, como ejemplos, los realizados para los géneros $A s$ anthus (Turner 2014), Axiniphyllum (González-Zamora \& Villaseñor 2017), Bartlettina (Turner 2010b), Brickellia (Schilling et al. 2015), Chrysactinia (Villaseñor \& Redonda-Martínez 2009), Cosmos (Vargas-Amado et al. 2013, Castro-Castro et al. 2014), Dahlia (Castro-Castro et al. 2012), Florestina (Soto-Trejo et al. 2016), Lepidaploa (Redonda-Martínez \& Villaseñor 2011a), Melampodium (Stuessy et al. 2011), Sclerocarpus (Villaseñor \& Hinojosa-Espinosa 2011) o Zaluzania (Turner (2012b). Otros trabajos importantes de mencionar son los tratamientos florístico-taxonómicos realizados en algunos estados o regiones como parte de los proyectos florísticos en curso; tal es el caso de los fascículos de la Flora de Chiapas (Strother 1999), Flora del Bajío y de regiones adyacentes (e.g., García-López \& Koch 1995, Villarreal-Quintanilla et al. 2006, Rzedowski \& Calderón 2008, 2011, Redonda-Martínez 2016), Flora Novo-Galiciana (McVaugh 1984), Flora del Valle de Tehuacán-Cuicatlán (e.g., Villarreal-Quintanilla et al. 2008b, Redonda-Martínez \& Villaseñor 2009, 2011b, Medina-Lemos \& Villaseñor 2010) o Flora de Veracruz (e.g., Villarreal-Quintanilla \& Villaseñor 2004, Villarreal-Quintanilla et al. 2008a).

El objetivo de este trabajo es analizar la distribución de las especies de Asteraceae en México, evaluando la riqueza total conocida y el número de endemismos. Para ello se comparan valores a dos diferentes escalas: i) división de México por estados y ii) por bioma. Además, se pretende identificar áreas importantes para realizar futuras prospecciones florísticas, así como grupos taxonómicos que requieren de trabajo taxonómico particular y que futuros taxónomos en la familia podrían considerar como tema de estudio

\section{Materiales y métodos}

La fuente principal de análisis, además de la revisión de la amplia literatura florístico-taxonómica que trata sobre la familia Asteraceae en México (discutidas líneas atrás), es la base de datos que se ha venido generando de la revisión de material herborizado resguardado en más de 20 herbarios, tanto nacionales, como del extranjero (Villaseñor, datos no publicados). La lista actualizada de los géneros y especies de Asteraceae en México fue recientemente publicada (Villaseñor 2016), la cual es la base de los análisis que aquí se presentan. Se remite al lector a dicha fuente para revisar los nombres científicos de los taxa analizados.

Una especie es considerada endémica de México si solamente se ha registrado dentro de sus límites políticos y dentro de éstas, microendémica si no se tienen reportes de su existencia más allá del límite político de un estado. Por otra parte, para definir la presencia de las especies en los cinco principales biomas de México (Villaseñor \& Ortiz 2014), los sitios georreferenciados de recolecta se sobrepusieron a un mapa de biomas incluidos en formato digital dentro de un Sistema de Información Geográfica (ArcGIS 10.0, ESRI 2010). De esta manera fue posible cuantificar los números de especies en cada uno de los biomas que se usaron para evaluar los patrones de riqueza de especies de la familia.

\section{Resultados}

Diversidad taxonómica. La Tabla 1 muestra un resumen de la riqueza de géneros y especies de Asteraceae en México distribuidas por tribu. Destacan por su número las tribus Heliantheae, Eupatorieae, Astereae y Senecioneae. Las tribus Arctoteae y Calenduleae solamente están representadas por géneros adventicios (introducidos o exóticos), la primera por Arctotheca J.C. Wendl. y Gazania Gaertn. y la segunda por Calendula L. Los dos últimos géneros son ampliamente cultivados en muchas partes del país y sus reportes como miembros de la vegetación ruderal son escasos, probablemente derivados de desechos de material de jardines arrojados a las vías de comunicación; por el contrario, Arctotheca es de muy reciente identificación para la flora exótica nacional (Hinojosa-Espinosa \& Villaseñor 2015). Siete tribus registran más de 100 especies en México (Heliantheae, Eupatorieae, Astereae, Senecioneae, Coreopsideae, Mil- 
lerieae y Tageteae, Tabla 1). En conjunto, incluyen 2,219 especies, lo que representa $71 \%$ de la riqueza total. Es notable que se presenta el mismo orden de importancia respecto a sus especies endémicas, pues tales tribus también incluyen $82.8 \%$ (1,690 especies) del endemismo a nivel nacional.

La Tabla 2 detalla los géneros que cuentan con más de 20 especies. En conjunto incluyen 1,592 especies, 1,178 de ellas endémicas. Tales cifras representan la mitad (51.1\%) de la riqueza total y de endemismos de Asteraceae a nivel nacional (58.9\%); incluyen, igualmente, 275 especies microendémicas registradas hasta la fecha (Tabla 2). Ageratina y Stevia (Tribu Eupatorieae), así como Verbesina (Tribu Heliantheae), son géneros con más de 100 especies, la gran mayoría de ellas endémicas de México. De los géneros citados en la Tabla 2, prácticamente la mitad de sus especies son endémicas de México; entre las excepciones se cuentan Ambrosia (Heliantheae), Baccharis (Astereae), Fleischmannia (Eupatorieae) y Pseudognaphalium (Gnaphalieae).

Tabla 2. Géneros de Asteraceae con más de 20 especies en la flora de México. Endémicas incluye las especies endémicas de México y microendémicas a las endémicas restringidas a algún estado.

\begin{tabular}{|c|c|c|c|}
\hline Género & Especies & Endémicas & Microendémicas \\
\hline Ageratina & 167 & 135 & 41 \\
\hline Verbesina & 164 & 138 & 43 \\
\hline Stevia & 116 & 95 & 19 \\
\hline Erigeron & 87 & 65 & 16 \\
\hline Brickellia & 85 & 57 & 11 \\
\hline Acourtia & 76 & 67 & 14 \\
\hline Bidens & 68 & 43 & 15 \\
\hline Senecio & 63 & 43 & 6 \\
\hline Roldana & 55 & 43 & 4 \\
\hline Perymenium & 48 & 43 & 7 \\
\hline Psacalium & 47 & 46 & 8 \\
\hline Cirsium & 45 & 28 & 5 \\
\hline Pectis & 44 & 27 & 6 \\
\hline Perityle & 43 & 34 & 15 \\
\hline Melampodium & 40 & 22 & 2 \\
\hline Dahlia & 39 & 33 & 7 \\
\hline Aldama & 36 & 32 & 3 \\
\hline Pseudognaphalium & 33 & 12 & 2 \\
\hline Cosmos & 33 & 27 & 11 \\
\hline Baccharis & 31 & 12 & - \\
\hline Archibaccharis & 29 & 17 & 11 \\
\hline Ambrosia & 29 & 12 & 3 \\
\hline Tagetes & 27 & 15 & 2 \\
\hline Grindelia & 26 & 18 & 1 \\
\hline Tridax & 26 & 24 & 5 \\
\hline Koanophyllon & 23 & 18 & 5 \\
\hline Zinnia & 23 & 17 & 2 \\
\hline Bartlettina & 23 & 14 & 7 \\
\hline Critoniopsis & 23 & 19 & 2 \\
\hline Fleischmannia & 22 & 6 & - \\
\hline Coreopsis & 21 & 16 & 2 \\
\hline
\end{tabular}


El Apéndice 1 incluye los géneros de Asteraceae de la flora de México, ordenados alfabéticamente por tribus. Se indica para cada uno de ellos el número total de especies, de especies endémicas y de exóticas (cuando registran), así como una referencia bibliográfica que reporta la revisión taxonómica del género o alguna bibliografía útil para la identificación de sus especies.

Riqueza a nivel estatal. La Tabla 3 muestra la distribución de la riqueza de Asteraceae repartida entre los 32 estados en que políticamente se divide el país. Los cinco estados que destacan por su número de especies son Oaxaca, Jalisco, Durango, Guerrero y Michoacán; en el otro extremo, los estados con menor número de especies se ubican en la Península de Yucatán (Campeche, Quintana Roo y Yucatán).

Por su número de especies endémicas, los mismos estados con mayor riqueza total ocupan los primeros lugares. Un contraste se observa cuando se evalúan las especies microendémicas (aquellas restringidas a un solo estado), pues aunque Oaxaca permanece en primera posición,

Tabla 3. Riqueza de Asteraceae en cada uno de los 32 estados en que se divide políticamente México. Endémicas se refiere a especies endémicas de México y Microendémicas a endémicas restringidas al estado.

\begin{tabular}{|c|c|c|c|c|}
\hline Estado & Géneros & Especies & Endémicas & Microendémicas \\
\hline Aguascalientes & 133 & 379 & 192 & - \\
\hline Baja California & 141 & 366 & 89 & 33 \\
\hline Baja California Sur & 109 & 269 & 102 & 49 \\
\hline Campeche & 87 & 153 & 13 & - \\
\hline Chiapas & 198 & 656 & 137 & 27 \\
\hline Chihuahua & 194 & 782 & 329 & 8 \\
\hline Ciudad de México & 128 & 355 & 164 & - \\
\hline Coahuila & 185 & 672 & 280 & 23 \\
\hline Colima & 137 & 520 & 295 & 6 \\
\hline Durango & 212 & 909 & 516 & 17 \\
\hline Guanajuato & 159 & 577 & 314 & - \\
\hline Guerrero & 186 & 855 & 517 & 42 \\
\hline Hidalgo & 176 & 656 & 328 & 4 \\
\hline Jalisco & 197 & 956 & 607 & 33 \\
\hline Estado de México & 190 & 776 & 441 & 5 \\
\hline Michoacán & 181 & 837 & 499 & 10 \\
\hline Morelos & 145 & 505 & 257 & 3 \\
\hline Nayarit & 148 & 538 & 315 & -- \\
\hline Nuevo León & 175 & 631 & 279 & 7 \\
\hline Oaxaca & 210 & 1,040 & 600 & 139 \\
\hline Puebla & 197 & 759 & 399 & 12 \\
\hline Querétaro & 182 & 663 & 336 & - \\
\hline Quintana Roo & 81 & 148 & 10 & - \\
\hline San Luis Potosí & 208 & 775 & 377 & 5 \\
\hline Sinaloa & 161 & 594 & 331 & 10 \\
\hline Sonora & 194 & 681 & 249 & 10 \\
\hline Tabasco & 101 & 179 & 12 & - \\
\hline Tamaulipas & 198 & 640 & 270 & 11 \\
\hline Tlaxcala & 94 & 275 & 129 & - \\
\hline Veracruz & 221 & 834 & 367 & 18 \\
\hline Yucatán & 82 & 146 & 15 & - \\
\hline Zacatecas & 179 & 684 & 382 & 1 \\
\hline
\end{tabular}


Tabla 4. Riqueza de especies de Asteraceae en los cinco principales biomas de México. Entre paréntesis se indican las especies endémicas de México y restringidas al bioma.

\begin{tabular}{lcccc} 
Bioma & Géneros & Especies & Especies endémicas & Especies restringidas \\
\hline Bosque húmedo de montaña & 169 & 814 & 425 & $76(47)$ \\
Bosque templado & 266 & 1,906 & 1,302 & $746(656)$ \\
Bosque tropical húmedo & 145 & 365 & 107 & $67(29)$ \\
Bosque tropical estacionalmente seco & 184 & 811 & 474 & $140(127)$ \\
Matorral xerófilo & 283 & 1,254 & 615 & $486(226)$ \\
\hline
\end{tabular}

otros estados que no destacaban al comparar la riqueza total o el endemismo nacional, sobresalen al respecto. Entre ellos se cuentan los dos que constituyen el territorio de la península de Baja California, así como el estado de Coahuila.

Riqueza a nivel de biomas. Al evaluar los valores de riqueza de Asteraceae en los cinco principales biomas discutidos por Villaseñor \& Ortiz (2014), se observa que los bosques templados (BTEM) y los matorrales xerófilos (MXE) registran la mayor riqueza de especies y de endemismos (Tabla 4). Los bosques tropicales húmedos constituyen el bioma con la menor representación de miembros de esta familia, aunque un alto porcentaje de sus especies restringen allí su presencia.

Aunque la familia se caracteriza por tener especies que se cuentan entre las de mayor presencia en diversos hábitats, constituyendo malezas bastante conspicuas en muchos paisajes, es interesante notar que un alto porcentaje de sus miembros manifiestan una fidelidad ecológica a los ambientes de un bioma en particular. El número de especies conocidas hasta la fecha creciendo en un solo bioma (Tabla 4), constituye $49 \%$ de la riqueza total de la familia conocida en el país.

Esfuerzo de recolecta de Asteraceae en México y capital humano involucrado. La información discutida en este trabajo ha sido en gran parte obtenida del esfuerzo llevado a cabo por numerosos botánicos que han recolectado las especies conocidas de Asteraceae en México. La base de datos sobre las Compuestas mexicanas (Villaseñor, datos no publicados) reporta poco más de 200,000 ejemplares de esta familia, distribuidos en más de 20 colecciones botánicas, tanto nacionales como del extranjero. Aunque lejos de ser exhaustiva, dicha base de datos documenta una importante proporción de los especímenes recolectados para la familia en México, los cuales permiten hacer generalizaciones sobre patrones de riqueza y distribución como los discutidos en este trabajo. La Tabla 5 presenta, a manera de ejemplo, los 10 herbarios con mayor número de exsiccata revisados de la familia; destaca el Herbario Nacional de México (MEXU) del Instituto

\begin{tabular}{llc} 
Tabla 5. Los 10 herbarios con mayor número de especímenes de la familia & Asteraceae recolectados en \\
México, los cuales han sido revisados e identificados hasta especie por el autor. & & \\
Herbario & Siglas & Especímenes \\
\hline Herbario Nacional de México, Instituto de Biología, UNAM & MEXU & 113,317 \\
Universidad de Texas, Austin & LL y TEX & 50,627 \\
Instituto de Ecología A.C., Xalapa & XAL & 12,552 \\
Instituto de Ecología, Pátzcuaro & IEB & 11,598 \\
Herbario Luz María Villarreal de Puga, Universidad de Guadalajara & IBUG & 6,039 \\
Herbario Isidro Palacios, Instituto de Investigación de Zonas Desérticas, UASLP & SLPM & 6,027 \\
Escuela Nacional de Ciencias Biológicas, IPN & ENCB & 5,393 \\
Missouri Botanical Garden & MO & 4,263 \\
Herbario particular de la Familia Hinton, Coahuila & HINTON & 3,987 \\
Herbario Maestra Graciela Calderón y Jerzy Rzedowski, IPN, Durango & CIIDIR & 3,865
\end{tabular}


de Biología, UNAM. Entre los herbarios extranjeros con material revisado sobresalen los de la Universidad de Texas en Austin (LL y TEX) y el del Jardín Botánico de Missouri (MO).

Todo el material revisado y depositado en las colecciones botánicas (herbarios), proviene del esfuerzo de más de 7,000 recolectores documentados hasta la fecha. Sin embargo, unos 80 de ellos destacan por su importante participación en el esfuerzo de recolecta, con más de 400 números de Asteraceae; en la Tabla 6 se muestran igualmente, a manera de ejemplo, los recolectores con más de 1,000 números de Asteraceae dentro de su historial de recolecta de especímenes de la flora de México.

Asteraceae continúa siendo una de las familias con mayor atención por parte de los botánicos que trabajan sobre la flora del país, especialmente en cuanto a la exploración y recolección de sus miembros. Al ser una familia con numerosas especies, muchas de ellas muy ubicuas, no es de sorprender que la mayoría de los inventarios florísticos la reporten como dominante por su riqueza de especies. Una evaluación del número de registros de Asteraceae contenidos en una base de datos (Villaseñor, datos no publicados), registra casi 120,000 registros únicos identificados y georreferenciados; sumando duplicados de dichos registros, resguardados en más de 20 herbarios, tanto nacionales como del extranjero, se puede resumir que el conocimiento de la familia en México descansa en el estudio y revisión de más de 200,000 exsiccata (Figura 1).

Aunque la evaluación del conocimiento para la familia es bastante optimista, todavía se desconoce mucho acerca de la distribución geográfica de sus miembros ("Déficit Wallaceano") como se observa en la figura 1. Dividiendo México en cuadros de 6 minutos de latitud y longitud (aproximadamente $107 \mathrm{~km}^{2}$ de superficie), el número de cuadros sin un solo espécimen

Tabla 6. Recolectores de la flora de México con más de 1,000 números de Asteraceae identificados hasta especie.

\begin{tabular}{lc} 
Colector & Números de Asteraceae \\
\hline Jesús Balleza Cadengo & 5,706 \\
Jerzy Rzedowski & 4,694 \\
George B. Hinton & 4,598 \\
Dennis E. Breedlove & 3,165 \\
Pedro Tenorio Lezama & 2,773 \\
Eizi Matuda & 2,425 \\
José Carmen Soto Núñez & 2,179 \\
José Luis Villaseñor & 2,112 \\
Esteban Martínez Salas & 2,038 \\
Cyrus G. Pringle & 1,996 \\
Juan Ismael Calzada & 1,625 \\
Tetsukazu Yahara & 1,625 \\
Francisco Ventura Aburto & 1,519 \\
Rafael Hernández Magaña & 1,410 \\
Rafael Torres Colín & 1,359 \\
Rogers McVaugh & 1,309 \\
Oswaldo Téllez Valdés & 1,296 \\
James S. Henrickson & 1,281 \\
Robert M. King & 1,223 \\
Jorge Calónico Soto & 1,116 \\
Alush Shilom Ton & 1,099 \\
Guy L. Nesom & 1,072 \\
Tod F. Stuessy & 1,067 \\
Luz María Villarreal de Puga & 1,063 \\
Marshall C. Johnston & 1,032 \\
Arthur J. Cronquist & 1,019 \\
\hline &
\end{tabular}


Figura 1. Esfuerzo de recolecta de especímenes de la familia Asteraceae en México. A) Sitios de recolecta puntuales. B) Densidad de especímenes recolectados en celdas de $6 \times 6$ ' de latitud y longitud (aproximadamente $107 \mathrm{~km}^{2}$ ); los sitios marcados en rojo indican ausencia de recolecta de especímenes.
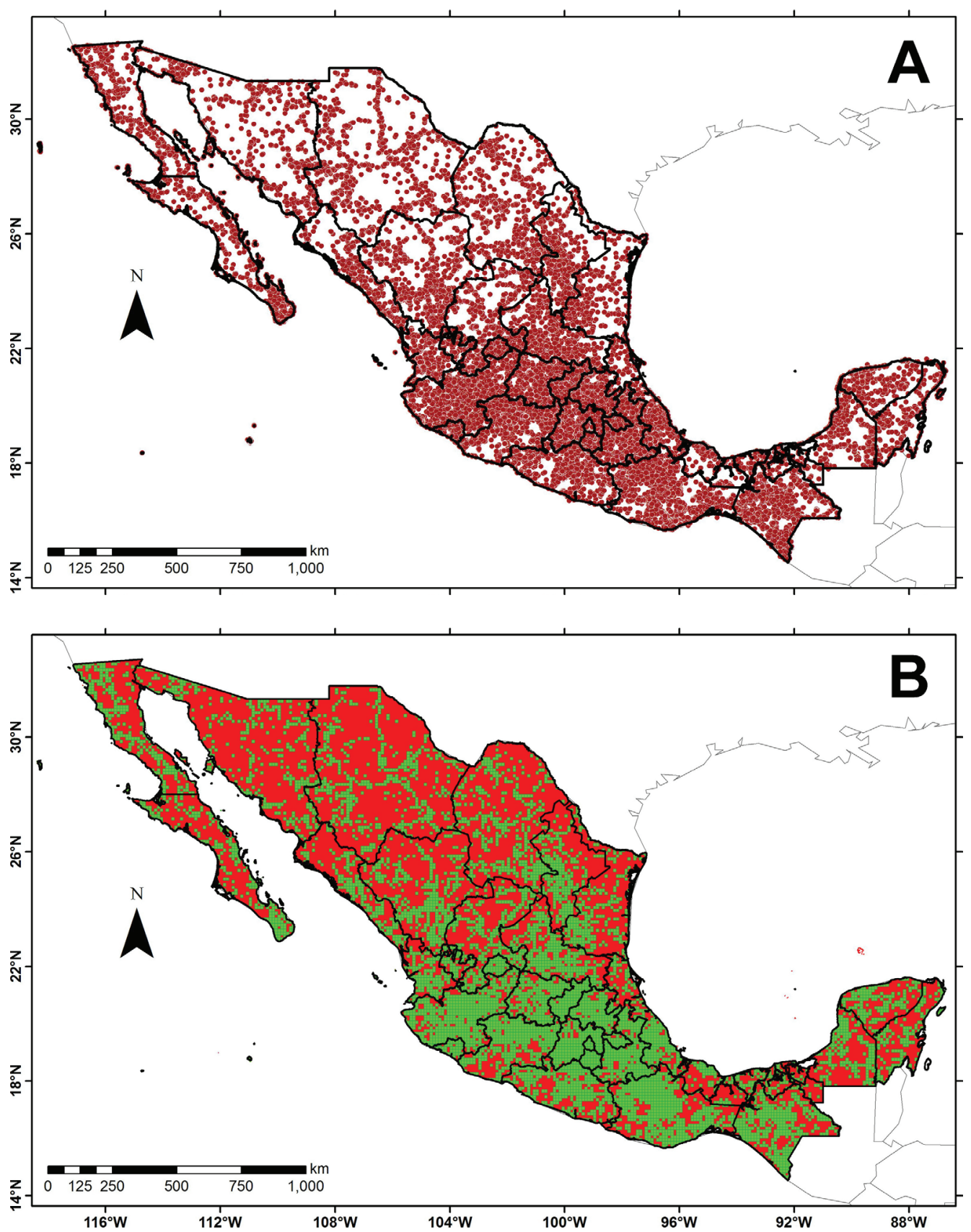

recolectado es mayor (10,655 de un total de 18,411 cuadros) a los que sí registran al menos un mínimo esfuerzo de recolecta $(57.9 \%$ sin registros, $42.1 \%$ con al menos un espécimen recolectado). Se puede observar que gran parte del territorio nacional, al norte del Trópico de Cáncer, está pobremente representado en las colecciones. Sin embargo, otras porciones del país, como la Península de Yucatán, la parte norte del Istmo de Tehuantepec o al sur en la vertiente del Pacífico (estados de Michoacán, Guerrero y Oaxaca), también contienen importantes extensiones donde no se han recolectado especímenes de la familia.

\section{Discusión}

En México están representadas, con miembros nativos, poco más de la mitad de las tribus en que actualmente se clasifica la familia (24 de un total de 45 ). Sobresalen por su número de es- 
pecies las tribus Heliantheae, Eupatorieae, Astereae y Senecioneae (Tabla 1). Otras tribus que actualmente contienen importantes cifras de especies, como Coreopsideae o Millerieae anteriormente formaban parte de tribus con circunscripciones diferentes (como Heliantheae), que la nueva sistemática filogenética las recupera como ensambles monofiléticos, con una posición y circunscripción alternativa (e.g., Baldwin 2009, Funk et al. 2009). Se requiere evaluar bajo estos nuevos criterios de clasificación a las diferentes tribus, para entender mejor su circunscripción, así como sus integrantes y analizar diferentes aspectos de su historia natural como, por ejemplo, las formas de crecimiento que incluyen, su variación morfológica, su distribución geográfica o los ambientes donde se les encuentra. Hasta la fecha, el único resumen en este sentido ha sido llevado a cabo para la tribu Vernonieae (Redonda-Martínez 2017).

Desde la revisión sobre la información taxonómica para la familia llevada a cabo por Cabrera-Rodríguez \& Villaseñor (1987), no se ha vuelto a realizar una síntesis sobre el conocimiento que se tiene sobre la sistemática de sus miembros. Sin embargo, la descripción de nuevos taxa (especies, géneros) o los cambios a nivel tribal, que como resultado de los estudios filogenéticos han ocurrido dentro de la familia, indican que el grupo constituye un campo fértil para que nuevos estudiantes interesados en estos temas consideren a la familia como una oportunidad de desarrollo profesional. La información mostrada en el Apéndice 1 revela que más del $70 \%$ de los géneros presentes en México han sido estudiados taxonómicamente en algún momento, o cuentan con las herramientas para la identificación de sus especies; desafortunadamente, muchos de esos estudios fueron hechos hace más de 30 años. En consecuencia, varios de ellos ya están obsoletos por la adición de nuevas especies a la flora o por los cambios taxonómicos derivados de los estudios sistemáticos. En consecuencia, es necesario evaluar para los géneros que cuentan con revisión taxonómica, la necesidad de actualizar su información, junto con los estudios pertinentes en aquellos que aún no cuentan con el estudio taxonómico correspondiente.

Además de los datos derivados de los estudios de biología molecular, actualmente se están explorando características consideradas como algo pasadas de moda, pero que, combinadas con los nuevos enfoques de estudio, enriquecen y robustecen más las hipótesis sobre relaciones filogenéticas y evolución de las especies mexicanas de Asteraceae. Como ejemplos se pueden citar los rasgos morfológicos estudiados en los aquenios (cipselas) u hojas de algunas especies (Redonda-Martínez et al. 2016, 2017, Rivera et al. 2017, Rojas-Leal et al. 2014, 2017) o las variaciones en los números cromosómicos (Watanabe et al. 2001, Soto-Trejo et al. 2013).

Los estados con mayor riqueza de especies de Asteraceae albergan también una amplia riqueza florística en general (por ejemplo, Chiapas, Jalisco, Oaxaca o Veracruz). Resultados similares se reportan para otros grupos vegetales, por ejemplo Apocynaceae (Juárez-Jaimes et al. 2007), Cucurbitaceae (Lira et al. 2002), Solanaceae (Martínez et al. 2017) o las lianas (Ibarra-Manríquez et al. 2015). Sin embargo, es notable destacar que otros estados no conocidos en particular por su notable riqueza florística, como Chihuahua, Coahuila o Durango, presentan un alto número de especies de Asteraceae y en especial de endemismos (Tabla 3). Estos últimos tres estados contienen grandes extensiones de bosques templados y de matorrales xerófilos, comunidades vegetales caracterizadas por contener importantes cifras de miembros de esta familia y en particular de endemismos (Rzedowski 1972, Tabla 4), los cuales han sido pobremente explorados (Figura 1). De igual manera, estados como Baja California, Baja California Sur o Guerrero sobresalen por su número de microendemismos (especies restringidas a su territorio) y seguramente, cuando sean visitados con mayor atención por los recolectores, indudablemente brindarán muchas sorpresas.

Un alto porcentaje de especies, tanto endémicas como no endémicas, constituyen rarezas ecológicas al conocerse solamente de un solo bioma (Tabla 4). Aunque varias de ellas sean reportadas como malezas, tanto arvenses como ruderales, es un hecho que su distribución, ya sea en ambientes primarios o transformados, se restringe a cierta fidelidad ambiental propia de cada uno de esos biomas, caracterizados por atributos climáticos y florístico-fisonómicos. Muchas de las especies de esta categoría deberían ser evaluadas con los criterios orientados a proveer información de su estado de vulnerabilidad. Varias de ellas necesitan ser incluidas bajo algún estado de riesgo en los esquemas actuales comprometidos con la conservación de la biodiversidad, como son las listas de la IUCN (2017) o la Norma Oficial Mexicana NOM-059 (SEMARNAT 2010). 
Entre las especies de Asteraceae reconocidas en la flora de México, se cuentan 63 que han sido introducidas (exóticas), formando parte de la flora de México como especies naturalizadas (Tabla 1). Villaseñor et al. (2012) hicieron un recuento de ellas y proporcionaron descripciones e imágenes como ayuda en su identificación. Sin embargo, se requieren estudios que ayuden a conocer mejor su comportamiento como malezas o malas hierbas y cómo influyen en las comunidades vegetales donde se les encuentra. Es importante subrayar que, aunque Asteraceae es la familia más diversa de la flora de México, ocupa el tercer lugar por su número de exóticas, superado en número por Poaceae y Fabaceae (Villaseñor \& Espinosa-García 2004).

En conclusión, la familia Asteraceae se revela como una familia de gran importancia en la flora de México, no nada más por sus niveles de riqueza y de endemismo. Es una familia que muestra una importante correlación con la riqueza florística total, por lo que es un buen subrogado de esta última (Villaseñor et al. 2005, 2007). Aunque no se ha cuantificado con precisión, observaciones personales sugieren que la familia es también una de las más abundantes en el sotobosque de los bosques templados o inclusive co-dominante en diversos tipos de matorrales xerófilos (por ejemplo, matorrales de Larrea-Flourensia o matorrales submontanos de Gochnatia).

Se espera que los datos aquí discutidos motiven a un mayor número de estudiantes a contribuir y estructurar un mejor marco de conocimiento biológico de esta importante familia de la flora de México. Todavía existen grandes espacios sin explorar (Figura 1), donde seguramente muchas novedades serán encontradas, varias de ellas como especies nuevas para la ciencia. Por otra parte, enfoques como los análisis de biodiversidad o la modelación de nichos ecológicos están revelando resultados novedosos desde diversos enfoques, que podrían aplicarse a regiones o grupos taxonómicos o a contestar preguntas de investigación específicas (Villaseñor 2015). Entre los ejemplos del potencial de estos nuevos enfoques se pueden citar los trabajos de Vargas-Amado et al. (2013), Villaseñor et al. (2013), Suárez-Mota et al. (2015a, b, 2016) o Estrada et al. (2016). Los miembros de Asteraceae se revelan cada vez mejor como excelentes objetos de estudio, motivando a un mayor número de especialistas en diversas áreas (anatomía, biogeografía, ecología, fitoquímica, etc.) a considerarlos como candidatos en sus investigaciones. Sin embargo, todavía se necesitan más interesados, especialmente jóvenes estudiantes que se involucren en algunos de los aspectos que todavía esperan ser estudiados para conocer mejor a esta importante familia de la flora de México.

\section{Agradecimientos}

La Comisión Nacional para el Conocimiento y Uso de la Biodiversidad (CONABIO) y el Instituto de Biología de la Universidad Nacional Autónoma de México (proyecto SIBA), apoyaron con recursos económicos y logísticos para la elaboración de la base de datos que documenta la riqueza florística de Asteraceae en México. Varios estudiantes, especialmente becarios del Consejo Nacional de Ciencia y Tecnología (CONACYT), han llevado actividades de entrenamiento tanto en la identificación de especímenes como en la georreferenciación de registros de la base de datos; a todos ellos se les agradece su entusiasmo e interés. Como siempre, sin la experimentada ayuda técnica del M. en C. Enrique Ortiz Bermúdez en el manejo de la información y en la elaboración de cuadros, figuras y mapas, la calidad de este trabajo dejaría mucho que desear. Este trabajo se dedica con mucho amor y agradecimiento a María del Pilar Sánchez, mi esposa, que, sin ser bióloga, ha compartido conmigo por más de 35 años el entusiasmo y gusto por la botánica y que sin su apoyo, este y muchos otros trabajos no se hubieran podido concretar. Se agradecen los comentarios de dos revisores anónimos que mejoraron substancialmente el contenido del manuscrito.

\section{Literatura citada}

Arriagada JE. 2003. Revision of the genus Clibadium (Asteraceae, Heliantheae). Brittonia 55: 245-301. DOI: 10.1663/0007-196X(2003)055[0245:ROTGCA]2.0.CO;2.

Baldwin BG. 2009. Heliantheae alliance. In: Funk VA, Sussana A, Stuessy TF, Bayer RJ, eds. 2009. Systematics, Evolution and Biogeography of the Compositae. Viena, Austria: International Association for Plant Taxonomy (IAPT). 
Balleza JJ, Villaseñor JL. 2011. Contribución del estado de Zacatecas (México) a la conservación de la riqueza florística del Desierto Chihuahuense. Acta Botanica Mexicana 94: 61-89.

Barkley TM, Janovec JP. 1996. Robinsonecio (Asteraceae-Senecioneae) a new genus from Mexico and Guatemala. SIDA 17: 77-81.

Bartoli A, Tortosa RD. 2012. Revision of the North American species of Grindelia (Asteraceae). Annals of the Missouri Botanical Garden 98: 447-513. DOI:10.3417/2008125.

Beaman JH. 1990. Revision of Hieracium (Asteraceae) in Mexico and Central America. Systematic Botany Monographs 29: 1-77. DOI.10.2307/25027732.

Bentham G. 1873. Notes on the classification, history, and geographical distribution of Compositae. Journal of the Linnean Society, Botany 13: 335-577. DOI.10.1111/j.1095-8339.1873.tb02575.x.

BFG [The Brazil Flora Group]. 2015. Growing knowledge: an overview of seed plant diversity in Brazil. Rodriguesia 66: 1-29. DOI: 10.1590/2175-7860201566411.

Bierner MW, Turner BL. 2003. Taxonomy of Tetraneuris (Asteraceae: Helenieae: Tetraneurinae). Lundellia 6: 44-96.

Bolick MR. 1991. Systematics of Salmea DC. (Compositae: Heliantheae). Systematic Botany 16: 462-477. DOI.10.2307/2419337.

Brako L, Zarucchi J. 1993. Catalogue of the flowering plants and gymnosperms of Peru. Monographs in Systematic Botany from the Missouri Botanical Garden 45: 1-1286.

Bremer K. 1987. Tribal interrelationships of the Asteraceae. Cladistics 3: 210-253. DOI: 10.1111/j.10960031.1987.tb00509.x.

Bremer K. 1994. Asteraceae. Cladistics and classification. Portland, Oregon: Timber Press.

Cabrera-Rodríguez L, Villaseñor JL. 1987. Revisión bibliográfica sobre el conocimiento de la familia Compositae en México. Biotica 12: 131-147.

Carlquist S. 1976. Tribal interrelationships and phylogeny of the Asteraceae. Aliso 8: 465-492.

Castro-Castro A, Rodríguez A, Vargas-Amado G, Harker M. 2012. Diversidad del género Dahlia (Asteraceae: Coreopsideae) en Jalisco, México y descripción de una especie nueva. Revista Mexicana de Biodiversidad 83: 347-358.

Castro-Castro A, Vargas-Amado G, Harker M, Rodríguez A. 2014. Análisis micromorfológico y citogenético del género Cosmos (Asteraceae, Coreopsideae), con una clave para su identificación. Botanical Sciences 92: 363-388. DOI: 10.17129/botsci.111.

Chambers KL. 1955. Biosystematic study of the annual species of Microseris. Contributions from the Dudley Herbarium. Stanford University.

Chambers KL. 1964. Nomenclature of Microseris lindleyi. Leaflets of Western Botany 10: 106-108.

Clark BL. 1999. Villasenoria (Asteraceae: Senecioneae): a new genus and combination from Mexico. SIDA 18: 631-635.

Cronquist A. 1950. A review of the genus Psilocarphus. Research Studies of the State College of Washington. 18: 71-89.

Cronquist A. 1981. An integrated system of classification of flowering plants. Nueva York: Columbia University Press. ISBN: 9780231038305

ESRI [Environmental Systems Research Institute]. 2010. ArcGIS 10.0. Redlands, California.

Estrada AS, Ortiz E, Villaseñor JL, Espinosa-García FJ. 2016. The distribution of cultivated species of Porophyllum (Asteraceae) and their wild relatives under climate change. Systematics and Biodiversity 14: 572-582. DOI: $10.1080 / 14772000.2016 .1205678$

Freeman CG, Barkley TM. 1995. A synopsis of the genus Packera (Asteraceae: Senecioneae) in Mexico. SIDA 16: 699-709.

Freire SE, Iharlegui L. 1997. Sinopsis preliminar del género Gamochaeta (Asteraceae, Gnaphalieae). Boletin de la Sociedad Argentina de Botánica 33: 23-35.

Funk VA, Kelloff C, Chan R. 2012. Phylogeny and biogeography of the tribe Liabeae (Composiae subfamily Cichorioideae). Taxon 61: 437-455.

Funk VA, Sancho G, Roque N. 2017. Nahuatlea: a new genus of Compositae (Gochnatieae) from North America. PhytoKeys 91: 105-124. DOI: 10.3897/phytokeys.91.21340.

Funk VA, Sussana A, Stuessy TF, Bayer RJ, eds. 2009. Systematics, Evolution and Biogeography of the Compositae. Viena, Austria: International Association for Plant Taxonomy (IAPT). ISBN: 978-39501754-3-1

Funston AM. 2008. Taxonomic revision of Roldana (Asteraceae: Senecioneae), a genus of the southwestern U.S.A., Mexico, and Central America. Annals of the Missouri Botanical Garden 95: 282-337. DOI: $10.3417 / 2003151$.

García-López E, Koch SD. 1955. Familia Compositae, Tribu Cardueae. Flora del Bajío y de Regiones Adyacentes. Instituto de Ecología, A. C., Centro Regional del Bajío. Pátzcuaro, Michoacán. 32: 1-51.

González-Zamora A, Villaseñor JL. 2017. Sinopsis del género Axiniphyllum (Asteraceae, Millerieae) endémico de México. Acta Botanica Mexicana 120: 121-129. DOI: 10.21829/abm120.2017.1214. 
Hansen H. 2004. Simplified key to four sections with 34 species in the genus Dahlia (Asteraceae-Coreopsideae). Nordic Journal of Botany 24: 549-553. DOI: 10.1111/j.1756-1051.2004.tb01639.x.

Heywood VH, Harborne JB, Turner BL, eds. 1977. The Biology and Chemistry of the Compositae. London: Academic Press. ISBN: 978-0123-468017

Hinojosa-Espinosa O, Villaseñor JL. 2015. Arctotheca prostrata (Asteraceae: Arctotideae), a South African species now present in Mexico. Botanical Sciences 93: 1-4. DOI: 10.17129/botsci.223.

Holmes WC. 1990. The genus Mikania (Compositae-Eupatorieae) in Mexico. SIDA, Botanical Miscellany 5: $1-45$.

Ibarra-Manríquez G, Rendón-Sandoval FJ, Cornejo-Tenorio G, Carrillo-Reyes P. 2015. Lianas of Mexico. Botanical Sciences 93: 365-417. DOI: 10.17129/botsci.123.

IUCN [International Union for Conservation of Nature]. 2017. The IUCN Red List of Threatened Species. Version 2017-3<www.iucnredlist.org> (consultada en julio 2017).

Jansen RK, Palmer JD. 1987. A chloroplast DNA inversion marks an ancient evolutionary split in the sunflower family (Asteraceae). Proceedings of the National Academy of Sciences of the United States of America 84: 5818-5822.

Jones SB. 1979. Taxonomic revision of Vernonia Section Leiboldia (Compositae, Vernonieae). Castanea 44: 229-237. DOI: $10.2307 / 4033181$

Juárez-Jaimes V, Alvarado-Cárdenas LO, Villaseñor JL. 2007. La familia Apocynaceae sensu lato en México: diversidad y distribución. Revista Mexicana de Biodiversidad 78: 459-482.

Katinas L, Gutiérrez DG, Grossi MA, Crisci JV. 2007. Panorama de la familia Asteraceae (=Compositae) en la República Argentina. Boletín de la Sociedad Argentina de Botánica 42: 113-129.

Keeley SC, Forsman ZH, Chan R. 2007. A phylogeny of the "evil tribe" (Vernonieae: Compositae) reveals Old/New World long distance dispersal: support from separate and combined congruent datasets (trnL-F, $n d h \mathrm{~F}, \mathrm{ITS})$. Molecular Phylogenetics and Evolution 44: 89-103. DOI: 10.1016/j.ympev.2006.12.024.

Kim K, Turner BL. 1992. Systematic overview of Krigia (Asteraceae-Lactuceae). Brittonia 44: 173-198. DOI: $10.2307 / 2806832$.

King RM, Robinson H. 1987. The genera of the Eupatorieae (Asteraceae). Monographs in Systematic Botany from the Missouri Botanical Garden 22: 1-581.

Lane MA, Hartman RL. 1996. Reclassification of North American Haplopappus (Compositae: Astereae) completed: Rayjacksonia gen. nov. American Journal of Boyany 83: 356-370. DOI: 10.1002/j.15372197.1996.tb12716.x.

Leppik EE. 1970. Evolutionary differentiation of the flower head of the Compositae II. Annales Botanici Fennici 7: 325-352.

Lira R, Villaseñor JL, Ortiz E. 2002. A proposal for the conservation of the family Cucurbitaceae in Mexico. Biodiversity and Conservation 11: 1699-1720. DOI: 10.1023/A:1020303905416.

Loockerman DJ, Turner BL, Jansen RK. 2003. Phylogenetic relationships within Tageteae (Asteraceae) based on nuclear ribosomal ITS and chloroplast $n d h F$ gene sequences. Systematic Botany 28: 191-207. DOI: 10.1043/0363-6445-28.1.191.

Martínez M, Vargas-Ponce O, Rodríguez A, Chiang F, Ocegueda S. 2017. Solanaceae family in Mexico. Botanical Sciences 95: 131-145. DOI: 10.17129/botsci.658.

McVaugh R. 1984. Compositae. Flora Novo-Galiciana. Volúmen 12. The University of Michigan Press. Ann Arbor.

Medina-Lemos R, Villaseñor JL. 2010. Asteraceae, Tribu Plucheeae. Flora del Valle de Tehuacán-Cuicatlán. Ciudad de México: Instituto de Biología, UNAM. 78: 1-13.

Melchert TE. 2010a. Bidens. In: Turner BL, ed. The Comps of Mexico. A Systematic account of the family Asteraceae (Chapter 10: Subfamily Coreopsideae). Phytologia Memoirs 15: 3-56.

Melchert TE. 2010b. Thelesperma. In: Turner BL, ed. The Comps of Mexico. A Systematic account of the family Asteraceae (Chapter 10: Subfamily Coreopsideae). Phytologia Memoirs 15: 130-137.

Morgan DR. 1993. A molecular systematic study and taxonomic revision of Psilactis (Asteraceae: Astereae). Systematic Botany 18: 290-308. DOI: 10.2307/2419405.

Morgan DR, Hartman RL. 2003. A synopsis of Machaeranthera (Asteraceae: Astereae), with recognition of segregate genera. SIDA 20: 1387-1416.

Murguía M, Villaseñor JL. 1996. GENCOMEX. Versión 1.0. Policlave para la identificación de los géneros de Compositae presentes en México. DF., México: Asociación de Biólogos Amigos de la Computación, A.C./Comisión Nacional para el Conocimiento y Uso de la Biodiversidad. <www.abatax. abacoac.org/clavesTax_lista.php> (Consultado octubre de 2017).

Nesom GL. 1990a. Taxonomy of Achyrocline (Asteraceae: Inuleae) in Mexico and Central America. Phytologia 68: 181-185.

Nesom GL. 1990b. Taxonomy of Gnaphaliothamnus (Asteraceae: Inuleae). Phytologia 68: 366-381.

Nesom GL. 1990c. Taxonomy of the genus Laennecia (Asteraceae: Astereae). Phytologia 68: 205-228. 
Nesom GL. 1990d. Mexerion (Asteraceae: Inuleae), a new genus from Mexico. Phytologia 68: 247-254.

Nesom GL. 1991a. Taxonomy of Isocoma (Compositae: Astereae). Phytologia 70: 69-114.

Nesom GL. 1991b. Tomentaurum (Asteraceae: Astereae), a new genus of goldenaster from Chihuahua, México. Phytologia 71: 128-131.

Nesom GL. 1993. Aztecaster (Asteraceae: Astereae), a new ditypic genus of dioecious shrubs from Mexico with redefinitions of the Subtribes Hinterhuberinae and Baccharidinae. Phytologia 75: 55-73.

Nesom GL. 1994. Review of the taxonomy of Aster sensu lato (Asteraceae: Astereae), emphasizing the New World species. Phytologia 77: 141-297.

Nesom GL. 1997. Synopsis of Stephanodoria sensu lato (Asteraceae: Astereae). Phytologia 82: 107-113.

Nesom GL, Baird GI. 1993. Completion of Ericameria (Asteraceae: Astereae), diminution of Chrysothamnus. Phytologia 75: 74-93.

Nesom GL, Noyes R.D. 2000. Batopilasia (Asteraceae: Astereae), a new genus from Chihuahua, Mexico. SIDA 79: 79-84.

Nesom GL, Youngbae S, Morgan DR, Sundberg SD y Simpson BB. 1991. Chloracantha a new genus of North American Astereae (Asteraceae). Phytologia 70: 371-381.

Panero JL, Villaseñor JL. 1996. Tehuana calzadae (Asteraceae: Heliantheae) gen. et sp. nov. from the Pacific coast of Oaxaca, Mexico. Systematic Botany 21: 553-557. DOI: 10.2307/2419614.

Pelser PB, Nordenstam B, Kadereit JW, Watson LE. 2007. An ITS phylogeny of tribe Senecioneae (Asteraceae) and a new delimitation of Senecio L. Taxon 56: 1077-1104. DOI: 10.2307/25065905.

Pruski JF. 2016. Compositae of Central America-IV. The genus Eremosis (Vernonieae), non-glandular trichomes and pericarp crystals. Phytoneuron 2016-50: 1-41.

Pruski JF. 2017. Compositae of Central America-VIII. The genus Lepidaploa (Vernonieae. Phytoneuron 2017-50: 1-39.

Pruski JF, Hartman RL. 2012. Synopsis of Leucosyris, including synonymous Arida (Compositae: Astereae). Phytoneuron 2012-98: 1-15.

Pruski JF, Tadesse M, Crawford DJ. 2015. Studies of Neotropical Compositae-XI. The new generic name Electranthera (Coreopsideae). Phytoneuron 2015-68: 1-17.

Rangel-Churio JO. 2015. La riqueza de las plantas con flores de Colombia. Caldasia 37: 279-307. DOI: 10.15446/caldasia.v37n2.54375.

Redonda-Martínez R. 2016. Familia Compositae, Tribu Inuleae. Flora del Bajío y de Regiones Adyacentes. Pátzcuaro, Michoacán Instituto de Ecología, A.C., Centro Regional del Bajío. 194: 1-13.

Redonda-Martínez R. 2017. Diversidad y distribución de la tribu Vernonieae (Asteraceae) en México. Acta Botanica Mexicana 119: 115-138. DOI: 10.21829abm119.2017.1235.

Redonda-Martínez R, Terrazas T, Rojas-Leal A, Villaseñor JL. 2017. The morpho-anatomy of cypselae in sutribe Leiboldiinae (Vernonieae, Asteraceae). Brazilian Journal of Botany 40: 491-502. DOI: 10.1007/s40415-016-0349-9.

Redonda-Martínez R, Villaseñor JL. 2009. Asteraceae, Tribu Vernonieae. Flora del Valle de TehuacánCuicatlán. Ciudad de México: Instituto de Biología, UNAM. 72: 1-23.

Redonda-Martínez R, Villaseñor JL. 2011a. El género Lepidaploa (Familia Asteraceae, Tribu Vernonieae) en México. Revista Mexicana de Biodiversidad 82: 782-797.

Redonda-Martínez R, Villaseñor-Ríos. 2011b. Asteraceae, Tribu Senecioneae. Flora del Valle de Tehuacán-Cuicatlán. México: Instituto de Biología, UNAM. 89: 1-64.

Redonda-Martínez R, Villaseñor JL, Terrazas T. 2016. Trichome diversity in the subtribe Leiboldiinae (Vernonieae, Asteraceae). Journal of the Torrey Botanical Society 143: 298-310. DOI: 10.3159/TORREY-D-14-00062.1.

Rivera P, Villaseñor JL, Terrazas T. 2017. Meso- or xeromorphic? Foliar characters of Asteraceae in a xeric scrub of Mexico. Botanical Studies 58: 12. DOI: 10.1186/s40529-017-0166-x.

Robinson H. 1978. Lundellianthus, a new genus from Guatemala (Heliantheae: Asteraceae). Wrightia 6: 40-43.

Robinson H. 1981. A revision of the tribal and subtribal limits of the Heliantheae (Asteraceae). Smithsonian Contributions to Botany 51: 1-102.

Robinson H. 1983. A generic review of the Tribe Liabeae (Asteraceae). Smithsonian Contributions to Botany 54: 1-67. DOI: 10.5479/si.0081024X.54.

Robinson H. 1991. A review of the genus Macvaughiella (Eupatorieae: Asteraceae) with two new species. Systematic Botany 16: 639-643. DOI: 10.2307/2418867.

Robinson H. 1992. A new genus Vernonanthura (Vernonieae, Asteraceae). Phytologia 73: 65-76. DOI: 105962/bhl.part.16736.

Robinson H, Brettell RD. 1973a. Studies in the Senecioneae (Asteraceae). I. A new genus, Pittocaulon. Phytologia 26: 451-453.

Robinson H, Brettell RD. 1973b. Studies in the Senecioneae (Asteraceae). II. A new genus, Nelsonianthus. Phytologia 27: 53-54. DOI: 10.5962/bhl.part.13906. 
Robinson H, Brettell RD. 1974. Studies in the Senecioneae (Asteraceae). V. The genera Psacaliopsis, Barkleyanthus, Telanthophora and Roldana. Phytologia 27: 402-439. DOI:10.5962/bhl.part.13922.

Robinson H, Cuatrecasas J. 1977. Notes on the genus and species limits of Pseudogynoxys (Greenm.) Cabrera (Asteraceae: Senecioneae). Phytologia 36: 177-192.

Robinson H, Cuatrecasas J. 1978. A review of the Central American species of Pentacalia (Asteraceae: Senecioneae). Phytologia 40: 37-50.

Robinson H, Funk VA. 1987. A phylogenetic analysis of Leiboldia, Lepidonia, and a new genus Stramentopappus (Vernonieae: Asteraceae). Botanische Jahrbücher für Systematik, Pflanzengeschichte und Pflanzengeographie 108: 213-228.

Rojas-Leal A, Villaseñor JL, Terrazas T. 2014. Desarrollo del patrón de venación en cuatro especies de la tribu Senecioneae (Asteraceae). Botanical Sciences 92: 23-36. DOI: 10.17129/botsci.25.

Rojas-Leal A, Villaseñor JL, Terrazas T. 2017. Tricomas foliares en Senecio sección Mulgediifolii (Senecioneae, Asteraceae). Acta Botanica Mexicana 119: 69-78. DOI: 10.21829/abm1 19.2017.1232.

Rzedowski J. 1972. Contribuciones a la fitogeografía florística e histórica de México III. Algunas tendencias en la distribución geográfica y ecológica de las Compositae mexicanas. Ciencia (México) 27: 123-132.

Rzedowski J. 1978. Claves para la identificación de los géneros de la familia Compositae en México. Acta Científica Potosina 7: 5-145.

Rzedowski J. 1991. Diversidad y orígenes de la flora fanerogámica de México. Acta Botanica Mexicana 14: 3-21. DOI: 10.21829/abm14.1991.611.

Rzedowski J, Calderón de R G. 2008. Familia Compositae, Tribu Heliantheae I (géneros Acmella-Jefea). Flora del Bajio y de Regiones Adyacentes. Pátzcuaro, Michoacán: Instituto de Ecología, A.C., Centro Regional del Bajío. 157: 1-344.

Rzedowski J, Calderón de R G. 2011. Familia Compositae, Tribu Heliantheae II (géneros Lagascea-Zinnia). Flora del Bajío y de Regiones Adyacentes. Pátzcuaro, Michoacán: Instituto de Ecología, A.C., Centro Regional del Bajío. 172: 1-409.

Saroyan JP, Parnell DR, Strother JL. 2000. Revision of Corethrogyne (Compositae: Astereae). Madroño 47: 89-96.

Sauck JR. 1975. Distribution, chromosomes, and taxonomy of Perthenice mollis (Compositae). Madroño 23: 227-234.

Schilling EE. 1990. Taxonomic revision of Viguiera subg. Bahiopsis (Asteraceae: Heliantheae). Madroño 37: 149-170.

Schilling EE. 2008. Paneroa, a new genus of Eupatorieae (Asteraceae) from Mexico. Novon 18: 520-523. DOI: $10.3417 / 2007173$.

Schilling EE, Panero JL. 2002. A revised classification of subtribe Helianthinae (Asteraceae: Heliantheae) I. Basal lineages. Botanical Journal of the Linnean Society 140: 65-76. DOI: 10.1046/j.10958339.2002.00079.x.

Schilling EE, Panero JL. 2010. Transfers to Simsia and desription of Davilanthus, a new genus of Asteraceae. Brittonia 62: 309-320. DOI: 10.1007/s12228-010-9129-1.

Schilling EE, Panero JL. 2011. A revised classification of subtribe Helianthinae (Asteraceae: Heliantheae) II. Derived lineages. Botanical Journal of the Linnean Society 167: 311-331. DOI: 10.1111/j.10958339.2011.01172.x.

Schilling EE, Panero JL, Crozier BS, Scott RW, Dávila P. 2015. Bricklebush (Brickellia) phylogeny reveals dimensions of the great Asteraceae radiation in Mexico. Molecular Phylogenetics and Evolution 85: 161-170. DOI: 10.1016/j.ympev.2015.02.007.

SEMARNAT [Secretaría del Medio Ambiente y Recursos Naturales]. 2010. Norma Oficial Mexicana NOM-059-SEMARNAT-2010, Protección ambiental - Especies nativas de México de flora y fauna silvestres - Categorías de riesgo y especificaciones para su inclusión, exclusión o cambio - Lista de especies en riesgo._Diario Oficial de la Federación_.2da Sección, 30 de diciembre de 2010.

Semple JC, Bowers FD. 1985. A revision of the goldenaster genus Pityopsis Nutt. (Compositae: Astereae). University of Waterloo Biology Series 29: 1-34.

Sieren DJ. 1981. The taxonomy of the genus Euthamia. Rhodora 83: 551-579.

Small J. 1917. The origin and development of the Compositae. New Phytologist 16: 157-177. DOI: 10.1111/j.1469-8137.1917.tb07238.x.

Smith EB. 1989. A biosystematic study and revision of the genus Coreocarpus (Compositae). Systematic Botany 14: 448-472. DOI: 10.2307/2418990.

Soto-Trejo F, Palomino G, Villaseñor JL, Crawford DJ. 2013. Polyploidy in Asteraceae of the xerophytic scrub of the Ecologica Reserve of the Pedregal of San Angel, Mexico City. Botanical Journal of the Linnean Society 173: 211-229. DOI: 10.1111/boj.12080.

Soto-Trejo F, Schilling EE, Oyama K, Lira R, Dávila P. 2016. A taxonomic revision of the genus Florestina (Asteraceae, Bahieae). Phytotaxa 268: 91-109. DOI: 10.11646/phytotaxa.268.2.1. 
Spooner DM. 1990. Systematics of Simsia (Compositae-Heliantheae). Systematic Botany Monographs 30: 1-90. DOI: $10.2307 / 25027790$.

Strother JL. 1986. Renovation of Dyssodia (Compositae-Tageteae). SIDA 11: 371-378.

Strother JL. 1987. Damnxanthodium (Compositae: Heliantheae), a new genus from Mexico. Systematic Botany 12: 41-43. DOI: $10.2307 / 2419213$.

Strother JL. 1989. Expansion of Lundellianthus (Compositae: Heliantheae). Systematic Botany 14: $544-$ 548. DOI: $10.2307 / 2418999$.

Strother JL. 1991. Taxonomy of Complaya, Elaphandra, Iogeton, Jefea, Wamalchitamia, Wedelia, Zexmenia, and Zyzyxia (Compositae-Heliantheae-Ecliptinae). Systematic Botany Monographs 33: 1-111. DOI: $10.2307 / 25027802$.

Strother JL. 1999. Compositae-Heliantheae s.l. Flora of Chiapas Part 5. The California Academy of Sciences. Berkeley.

Strother JL, Pilz GE. 1975. Taxonomy of Psathyrotes (Compositae: Senecioneae). Madroño 23: 24-40.

Stuessy TF, Blöch C, Villaseñor JL, Rebernig CA, Weiss-Schneeweiss H. 2011. Phylogenetic analyses of DNA sequences with chromosomal and morphological data confirm and refine sectional and series classification within Melampodium (Asteraceae, Millerieae). Taxon 60: 436-449.

Suárez-Mota ME, Villaseñor JL. 2011. Las Compuestas endémicas de Oaxaca, México: diversidad y distribución. Boletín de la Sociedad Botánica de México 88: 55-66. DOI: 10.17129/botsci.308.

Suárez-Mota ME, Villaseñor JL, López-Mata L. 2015a. La región del Bajío, México y la conservación de su diversidad florística. Revista Mexicana de Biodiversidad 86: 799-808. DOI: 10.1016/j.rmb.2015.06.001 1870-3453

Suárez-Mota ME, Villaseñor JL, López-Mata L. 2015b. Ecological niche similarity between congeneric Mexican plant species. Plant Ecology and Evolution 148: 318-328. DOI: 10.5091/placevo.2015.1147.

Suárez-Mota ME, Ortiz E, Villaseñor JL, Espinosa-García FJ. 2016. Ecological niche modeling of invasive plants species according to invasion status and management needs: the case of Chromolaena odorata in South Africa. Polish Journal of Ecology 64: 369-383. DOI: 10.3161/15052249PJE2016.64.3.007.

Suárez-Mota ME, Villaseñor JL, López-Mata L. 2017. Dominios climáticos de la Sierra Madre Oriental y su relación con la diversidad florística. Revista Mexicana de Biodiversidad 88: 224-233. DOI: 10.1016/ j.rmb.2017.01.020.

Tanowitz BD. 1982. Taxonomy of Hemizonia sect. Madiomeris (Asteraceae: Madiinae). Systematic Botany 7: 314-339. DOI: $10.2307 / 2418392$.

Tomb AS. 1972. Re-establishment of the genus Prenanthella Rydb. (Compositae: Cichorieae). Brittonia 24: 223-228. DOI: $10.2307 / 2805872$.

Tomb AS. 1980. Taxonomy of Lygodesmia (Asteraceae). Systematic Botany Monographs 1: 1-51. DOI: $10.2307 / 25027585$.

Turner BL. 1984. Update on the genus Jaegeria (Compositae-Heliantheae). Phytologia 55: 243-251.

Turner BL. 1988. Taxonomic study of Chrysanthellum (Asteraceae: Coreopsideae). Phytologia 64: 410444. DOI: 10.5962/bhl.part.29749.

Turner BL. 1989. Revisionary treatment of the genus Sinclairia, including Liabellum (Asteraceae, Liabeae). Phytologia 67: 168-206. DOI.10.5962/bhl.part.26149.

Turner BL. 1990a. A reevaluation of the genus Alepidocline (Asteraceae, Heliantheae, Galinsoginae) and description of a new species from Oaxaca, Mexico. Phytologia 69: 387-392.

Turner BL. 1990b. An overview of the Mexican genus Digitacalia (Asteraceae, Senecioneae). Phytologia 69: 150-159. DOI: 10.5962/bhl.part.17992.

Turner BL. 1990c. Taxonomy of Varilla (Asteraceae: Heliantheae). Phytologia 68: 4-13. DOI: 10.5962/ bhl.part.19644.

Turner BL. 1994. Systematic study of the genus Eupatoriastrum (Asteraceae, Eupatorieae). Plant Systematics and Evolution 190: 113-127. DOI: 10.1007/BF00937862.

Turner BL. 1995a. Synopsis of Ageratella (Asteraceae: Eupatorieae). Phytologia 78: 204-208.

Turner BL. 1995b. Resubmergence of Hydrodyssodia B.L. Turner into Hydropectis McVaugh (Asteraceae, Tageteae) with description of a new species, Hydropectis estradii, from Chihuahua, Mexico. Phytologia 78: 211-213.

Turner BL. 1996. The Comps of Mexico. A Systematic account of the family Asteraceae. Volume 6. Tageteae and Anthemideae. Phytologia Memoirs 10: 1-93.

Turner BL. 1997. The Comps of Mexico. A Systematic account of the family Asteraceae. Volume 1. Eupatorieae. Phytologia Memoirs 11: 1-272.

Turner BL. 2000. Plateilema (Asteraceae: Helenieae) a new generic report for the United States. SIDA 19: $185-187$

Turner BL. 2005. A recension of the Mexican species of Roldana (Asteraceae: Senecioneae). Phytologia 87: 204-249. 
Turner BL. 2009a. Three new species of Koanophyllon (Asteraceae: Eupatorieae) from Mexico. Phytologia 91: 312-324.

Turner BL. 2009b. The Comps of Mexico. A Systematic account of the family Asteraceae. (Chapter 9: Subfamily Mutisioideae). Phytologia Memoirs 14: 1-129.

Turner BL. 2010a. Recension of the Mexican Amauriopsis (Asteraceae: Bahieae). Phytoneuron 2010-10: 1-7.

Turner BL. 2010b. Mexican species of the genus Bartlettina (Asteraceae: Eupatorieae), and erection of three new species. Phytologia 92: 279-303.

Turner BL. 2010c. The Comps of Mexico. A Systematic account of the family Asteraceae (Chapter 10: Subfamily Coreopsideae). Phytologia Memoirs 15: 1-224.

Turner BL. 2011. A new species of Decachaeta (Asteraceae: Eupatorieae), from Oaxaca, Mexico. Phytologia 93: 346-350.

Turner BL. 2012a. A new species of Achyropappus (Asteraceae: Bahieae) from Querétaro, Mexico. Phytoneuron 2012-83: 1-5.

Turner BL. 2012b. Recension of the Mexican species of Zaluzania (Asteraceae: Heliantheae). Phytologia 94: 319-333.

Turner BL. 2013a. A new species of Heliomeris (Asteraceae: Heliantheae) from Oaxaca, Mexico. Phytologia 95: 103-106.

Turner BL. 2013b. Recension of the Mexican species of Hymenostephium (Asteraceae: Heliantheae). Phytologia 95: 1-9.

Turner BL. 2013c. Recension of Mexican species of Otopappus (Asteraceae: Heliantheae). Phytologia 95: 151-160.

Turner BL. 2014. The genus Asanthus (Asteraceae: Eupatorieae) revisited. Phytologia 96: 195-198.

Turner BL. 2015. Recension of Viguiera (sensu stricto) (Asteraceae: Heliantheae). Phytologia 97: 16-24.

Turner BL, Dawson D. 1980. Taxonomy of Tetragonotheca (Asteraceae-Heliantheae). SIDA 8: 296-303.

Turner BL, Kim KJ. 1990. An overview of the genus Pyrrhopappus (Asteraceae: Lactuceae) with emphasis on the chloroplast DNA restriction site data. American Journal of Botany 77: 845-850. DOI: 10.1002/j.1537-2197.1990.tb15178.x.

Turner BL, Sundberg SD. 1986. Systematic study of Osbertia (Asteraceae-Astereae). Plant Systematics and Evolution 151: 229-239.

Turner BL, Watson T. 2007. Taxonomic revision of Gaillardia (Asteraceae). Phytologia Memoirs 13: 1-112.

Turner BL, Zippin D. 1992. Taxonomic sudy of Venegasia (Asteraceae: Helenieae). SIDA 15: 223-229.

Turner MW. 1993. Systematic study of the genus Baileya (Asteraceae: Helenieae). SIDA 15: 491-508.

Urbatsch LE, RP Roberts. 2004. New combinations in the genus Gundlachia and four new genera of Astereae (Asteraceae) from northern Mexico and the southern United States. SIDA 21: 243-257.

Van Horn GS. 1973. The taxonomic status of Pentachaeta and Chaetopappa with a revision of Pentachaeta. University of California Publications in Botany 65: 1-41.

Vargas-Amado G, Castro-Castro A, Harker M, Villaseñor JL, Ortiz E, Rodríguez A. 2013. Distribución geográfica y riqueza del género Cosmos (Asteracae: Coreopsideae). Revista Mexicana de Biodiversidad 84: 536-555. DOI.10.7550/rmb.31481.

Villarreal-Quintanilla JA, Villaseñor JL. 2004. Familia Compositae, Tribu Tageteae. Flora de Veracruz. Xalapa, Veracruz- Riverside, California: Instituto de Ecología, A.C. y University of California. 135: 1-67.

Villarreal-Quintanilla JA, Villaseñor JL, Medina L R. 2006. Familia Compositae, Tribu Helenieae. Flora del Bajío y de Regiones Adyacentes. Pátzcuaro, Michoacán: Instituto de Ecología, A.C., Centro Regional del Bajío. 140: 1-54.

Villarreal-Quintanilla JA, Villaseñor JL, Medina L R. 2008a. Familia Compositae, Tribu Helenieae. Flora de Veracruz. Xalapa, Veracruz: Instituto de Ecología, A.C. y Centro de Investigaciones Tropicales (CITRO), Universidad Veracruzana. 143: 1-70.

Villarreal-Quintanilla JA, Villaseñor-Ríos JL, Medina-Lemos R. 2008b. Asteraceae, Tribu Tageteae. Flora del Valle de Tehuacán-Cuicatlán. México: Instituto de Biología, UNAM. 62: 1-59.

Villaseñor JL. 1989. Manual para la identificación de las Compositae de la península de Yucatán y Tabasco. Technical Report 4. Claremont: Rancho Santa Ana Botanical Garden. DOI: 10.21829/ abm7.1989.1123.

Villaseñor JL. 1990. The genera of Asteraceae endemic to Mexico and adjacent regions. Aliso 12: 685 692. DOI: 10.5642/aliso.19901204.04.

Villaseñor JL. 1993. La Familia Asteraceae en México. Revista de la Sociedad Mexicana de Historia Natural. Volumen Especial XLIV: 117-124.

Villaseñor JL. 2015. ¿La crisis de la biodiversidad es la crisis de la taxonomía? Botanical Sciences 93: 1-12. DOI.10:17129/botsci.456. 
Villaseñor JL. 2016. Checklist of the native vascular plants of Mexico. Revista Mexicana de Biodiversidad 87: 559-902. DOI: 10.1016/j.rmb.2016.06.017.

Villaseñor JL, Espinosa-García FJ. 2004. The alien flowering plants of Mexico. Diversity and Distributions 10: 113-123. DOI.10.1111/j1366-9516.2004.00059.x.

Villaseñor JL, Hinojosa-Espinosa O. 2011. El género Sclerocarpus (Asteraceae, Heliantheae) en México. Revista Mexicana de Biodiversidad 82: 51-61.

Villaseñor JL, Ibarra-Manríquez G, Ocaña D. 1998. Strategies for the conservation of Asteraceae in México. Conservation Biology 12: 1066-1075. DOI: 10.1046/j.1523-1739.1998.97171.x.

Villaseñor JL, Ibarra-Manríquez G, Meave JA, Ortiz, E. 2005. Higher taxa as surrogates of plant biodiversity in a megadiverse country. Conservation Biology 19: 232-238. DOI:10.1111/j.1523-1739.2005.00264.x.

Villaseñor JL, Maeda P, Rosell JA, Ortiz E. 2007. Plant families as predictors of plant biodiversity in Mexico. Diversity and Distributions 13: 871-876. DOI: 10.1111/j.1472-4642.2007.00385.x

Villaseñor JL, Ortiz E. 2014. Biodiversidad de las plantas con flores (División Magnoliophyta) en México. Revista Mexicana de Biodiversidad S85: S134-S142. DOI: 10.7550/rmb.31987.

Villaseñor JL, Ortiz E, Hinojosa-Espinosa O, Segura-Hernández G. 2012. Especies de la familia Asteraceae exóticas a la flora de México. D. F., México: SAGARPA, SENASICA, CONACOFI, IBUNAM y ASOMECIMA.

Villaseñor JL, Ortiz E, Cadena RJ, Estrada AS. 2013. Patrones de riqueza florística en el estado de Jalisco: la tribu Senecioneae (Asteraceae) como estudio de caso. Ibugana 4: 63-78.

Villaseñor JL, Redonda-Martínez MR. 2009. El género Chrysactinia (Familia Asteraceae, Tribu Tageteae) en México. Revista Mexicana de Biodiversidad 80: 29-37.

Villaseñor JL, Strother JL. 1989. Tuxtla, a new genus for Zexmenia pittieri (Compositae: Heliantheae). Systematic Botany 14: 529-540. DOI: 10.2307/2418997.

Villaseñor JL, Villarreal JA. 2006. El género Pluchea (familia Asteraceae, tribu Plucheeae) en México. Revista Mexicana de Biodiversidad 77: 59-65.

Watanabe K, Yahara T, Soejima A, Ito M. 2001. Mexican species of the genus Stevia (Eupatorieae, Asteraceae): chromosome numbers and geographical distribution. Plant Species Biology 16: 49-68. DOI: 10.1046/j.1442-1984.2001.00050.x.

Watson TJ. 1977. The taxonomy of Xylorhiza (Asteraceae-Astereae). Brittonia 29: 199-216. DOI: $10.2307 / 2805854$.

Weber WA. 1943. Vigethia, a new genus of Compositae based on Wyethia mexicana Watson. Madroño 7: $97-128$.

Wilken DH. 1975. A systematic study of Hulsea (Asteraceae). Brittonia 27: 228-244. DOI: $10.2307 / 2805894$.

Williams EW. 1957. The genus Malacothrix (Compositae). American Midland Naturalist 58: 494-512. DOI: $10.2307 / 2422630$. 
Apéndice 1.

Géneros de Asteraceae en la flora de México. Después del año de publicación del género, con diagonales

se indican primero el número total de especies, el número de especies endémicas y el número de especies introducidas (exóticas). La referencia corresponde a la revisión taxonómica o alguna publicación que ayuda en la identificación de las especies del género. Cuando la referencia indica Cabrera-Rodríguez y Villaseñor (1987) se remite al lector a dicha publicación para consultar la revisión taxonómica del género. Con asterisco $(*)$ se señalan los géneros introducidos a la flora de México.

Tribu Anthemideae

Anthemis L., 1753 1/0/1 (Villaseñor et al. 2012)

Achillea L., 1753 1/0/0 (Turner 1996)

*Argyranthemum Webb ex Sch. Bip., 1844 1/0/1 (Villaseñor et al. 2012)

Cotula L., 1753 3/0/2 (Turner 1996)

*Dendranthema (DC.) Des MouL., 1860 1/0/1 (Villaseñor et al. 2012)

*Glebionis Cass., 1826101 (Villaseñor et al. 2012)

*Leucanthemum Mill., 1754 3/0/3 (Villaseñor et al. 2012)

*Matricaria L., 1753 2/0/2 (Villaseñor et al. 2012)

*Soliva Ruiz \& Pav., 1794 2/0/2 (Villaseñor et al. 2012)

Sphaeromeria Nutt., 1841 1/1/0 (Turner 1996)

*Tanacetum L., 1753 1/0/1 (Villaseñor et al. 2012)

Tribu Arctotideae

*Arctotheca J.C. Wendl., 1798 1/0/1 (Hinojosa-Espinosa \& Villaseñor, 2015)

*Gazania Gaertn., 1791 1/0/1 (Villaseñor et al. 2012)

\section{Tribu Astereae}

Almutaster A. Löve \& D. Löve, 1982 1/0/0 (Nesom 1994)

Aphanostephus DC., 1836 3/0/0 (Cabrera-Rodríguez \& Villaseñor 1987)

Archibaccharis Heering, 1904 29/17/0 (Cabrera-Rodríguez \& Villaseñor 1987)

Astranthium Nutt., 1840 9/8/0 (Cabrera-Rodríguez \& Villaseñor 1987)

Aztecaster G.L. Nesom 1993 2/2/0 (Nesom 1993)

Baccharis L., 1753 31/12/0 (Cabrera-Rodríguez \& Villaseñor 1987)

Batopilasia G.L. Nesom \& Noyes 2000 1/1/0 (Nesom \& Noyes 2000)

*Bellis L., 1753 1/0/1 (Villaseñor et al. 2012)

Chaetopappa DC., 1836 6/2/0 (Cabrera-Rodríguez \& Villaseñor 1987)

Chihuahuana Urbatsch \& R.P. Roberts, 2004 1/1/0 (Urbatsch \& Roberts 2004)

Chloracantha G.L. Nesom, Y.B. Suh, D.R. Morgan \& B.B. Simpson, 1991 1/0/0 (Nesom et al. 1991)

Conyza Less., 1832 7/1/0

Corethrogyne DC., 1836 1/0/0 (Saroyan et al. 2000)

Dichaetophora A. Gray, 1849 1/0/0 (Cabrera-Rodríguez \& Villaseñor 1987)

Dieteria Nutt., 1840 2/0/0 (Morgan \& Hartman 2003)

Egletes Cass., 1817 2/0/0 (Cabrera-Rodríguez \& Villaseñor 1987)

Ericameria Nutt., 1840 12/2/0 (Nesom \& Baird 1993)

Erigeron L., 1753 87/65/0 (Cabrera-Rodríguez \& Villaseñor 1987)

Euthamia (Nutt.) Cass., 1825 1/0/0 (Sieren 1981)

Geissolepis B.L. Rob., 1892 1/1/0 (Cabrera-Rodríguez \& Villaseñor 1987)

Grindelia Willd., 1807 26/18/0 (Bartoli \& Tortosa 2012)

Gundlachia A. Gray, 1880 4/3/0 (Urbatsch \& Roberts 2004)

Gutierrezia Lag., 1816 13/6/0 (Cabrera-Rodríguez \& Villaseñor 1987)

Gymnosperma Less., 1832 1/0/0 (Cabrera-Rodríguez \& Villaseñor 1987)

Hazardia Greene, 1887 9/6/0 (Cabrera-Rodríguez \& Villaseñor 1987)

Heterotheca Cass., 1817 10/8/0 (Cabrera-Rodríguez \& Villaseñor 1987)

Isocoma Nutt., 1840 11/5/0 (Nesom 1991a)

Laennecia Cass., 18221250 (Nesom 1990c)

Lessingia Cham., 1829 1/0/0

Leucosyris Greene, 1897 8/2/0 (Pruski \& Hartman 2012)

Lorandersonia Urbatsch, R.P. Roberts \& Neubig, 2005 2/0/0 (Urbatsch \& Roberts 2004)

Machaeranthera Nees, 1832 2/0/0 (Morgan \& Hartman 2003)

Medranoa Urbatsch \& R.P. Roberts, 2004 1/1/0 (Urbatsch \& Roberts 2004)

Monoptilon Torr. \& A. Gray, 1844 1/0/0

Neonesomia Urbatsch \& R.P. Roberts, 2004 2/1/0 (Urbatsch \& Roberts 2004)

Oreochrysum Rydb., 1906 1/0/0

Oritrophium (Kunth) Cuatrec., 1961 2/2/0

Osbertia Greene, 1895 2/1/0 (Turner \& Sundberg 1986)

Pentachaeta Nutt., 1840 1/0/0 (Van Horn 1973)

Pityopsis Nutt. 1841 1/0/0 (Semple \& Bowers 1985) 
Apéndice 1.

Continúa.

Psilactis A. Gray, 1849 5/1/0 (Morgan 1993)

Rayjacksonia R.L., Hartm. \& M.A. Lane, 1996 1/0/0 (Lane \& Hartman 1996)

Solidago L., 1753 19/7/0

Stenotus Nutt., 1840 1/1/0

Stephanodoria Greene, 1895 1/1/0 (Nesom 1997)

Symphyotrichum Nees, 1832 17/4/1 (Nesom 1994)

Tomentaurum G.L. Nesom, 1991 1/1/0 (Nesom 1991b)

Townsendia Hook., 1834 2/1/0 (Cabrera-Rodríguez \& Villaseñor 1987)

Xanthisma DC., 1836 14/8/0 (Morgan \& Hartman 2003)

Xanthocephalum Willd., 1807 6/5/0 (Cabrera-Rodríguez \& Villaseñor 1987)

Xylorhiza Nutt., 1840 3/0/0 (Watson 1977)

Xylovirgata Urbatsch \& R.P. Roberts, 2004 1/1/0 (Urbatsch \& Roberts 2004)

\section{Tribu Bahieae}

Achyropappus Kunth, 1818 2/2/0 (Turner 2012a)

Amauriopsis Rydb., 1914 5/3/0 (Turner 2010a)

Bahia Lag., 1816 3/2/0 (Cabrera-Rodríguez \& Villaseñor 1987)

Bartlettia A. Gray, 1855 1/0/0

Chaetymenia Hook. \& Arn., 1841 1/1/0

Espejoa DC., 1836 1/0/0

Florestina Cass., 1817 8/5/0 (Soto-Trejo et al. 2016)

Hymenopappus L'Her., 1788 7/2/0 (Cabrera-Rodríguez \& Villaseñor 1987)

Hymenothrix A. Gray, 1849 4/2/0 (Cabrera-Rodríguez \& Villaseñor 1987)

Loxothysanus B.L. Rob., 1907 2/2/ 0 (Cabrera-Rodríguez \& Villaseñor 1987)

Palafoxia Lag., 1816 7/1/0 (Cabrera-Rodríguez \& Villaseñor 1987)

Peucephyllum A. Gray, 1859 1/0/0

Psathyrotopsis Rydb., 1927 3/2/0 (Strother \& Pilz 1975)

Schkuhria Roth, 1797 3/1/0 (Cabrera-Rodríguez \& Villaseñor 1987)

\section{Tribu Calenduleae}

${ }^{*}$ Calendula L., 1753 1/0/1 (Villaseñor et al. 2012)

\section{Tribu Cardueae}

*Carduus L., 1753 1/0/1 (Villaseñor et al. 2012)

*Carthamus L., 1753 1/0/1 (Villaseñor et al. 2012)

*Centaurea L., 1753 5/0/5 (Villaseñor et al. 2012)

Cirsium L., 1753 45/2/ 1 (Cabrera-Rodríguez \& Villaseñor 1987)

*Cynara L., 1753 1/0/1 (Villaseñor et al. 2012)

Plectocephalus D. Don, 1830 2/0/0 (Cabrera-Rodríguez \& Villaseñor 1987)

*Rhaponticum Ludw., 1757 1/0/1 (Villaseñor et al. 2012)

*Silybum Adans., 1763 1/0/1 (Villaseñor et al. 2012)

\section{Tribu Chaenactideae}

Chaenactis DC., 1836 8/1/0 (Cabrera-Rodríguez \& Villaseñor 1987)

\section{Tribu Cichorieae}

Agoseris Raf., 1817 1/0/0

Anisocoma Torr. \& A. Gray, 1844 1/0/0

Atrichoseris A. Gray, 1884 1/0/0

Calycoseris A. Gray, 1853 1/0/0

*Cichorium L., 1753 1/0/1 (Villaseñor et al. 2012)

Crepis L., 1753 2/0/1

*Hedypnois Mill., 1754 1/0/1 (Villaseñor et al. 2012)

*Helminthotheca Zinn., 1757 1/0/1 (Villaseñor et al. 2012)

Hieracium L., 1753 17/6/0 (Beaman 1990)

*Hypochaeris L., 1753 2/0/2 (Villaseñor et al. 2012)

Krigia Schreb., 1791 1/0/0 (Kim \& Turner 1992)

Lactuca L., $17537 / 1 / 2$

*Lapsana L., 1753 1/0/1 (Villaseñor et al. 2012)

*Leontodon L., 1753 1/0/1 (Villaseñor et al. 2012)

Lygodesmia D. Don, 1829 2/0/0 (Tomb 1980)

Malacothrix DC., 1838 10/2/0 (Williams 1957)

Marshalljohnstonia Henr., 1976 1/1/0 (Cabrera-Rodríguez \& Villaseñor 1987) 
Apéndice 1.

Continúa.

Microseris D. Don, 1832 2/0/0 (Chambers 1955)

Pinaropappus Less., 1832 10/7/0 (Cabrera-Rodríguez \& Villaseñor 1987)

Prenanthella Rydb., 1906 1/0/0 (Tomb 1972)

Pyrrhopappus DC., 1838 1/0/0 (Turner \& Kim 1990)

Rafinesquia Nutt., 1841 2/0/0

*Sonchus L., 1753 3/0/3 (Villaseñor et al. 2012)

Stebbinsoseris K.L. Chambers, 1991 1/0/0

Stephanomeria Nutt., 1841 9/2/0 (Cabrera-Rodríguez \& Villaseñor 1987)

*Taraxacum Weber, 1780 1/0/1 (Villaseñor et al. 2012)

*Tragopogon L., 1753 2/0/2 (Villaseñor et al. 2012)

Uropappus Nutt., 1841 1/0/0 (Chambers 1964)

*Youngia Cass., 1831 1/0/1 (Villaseñor et al. 2012)

\section{Tribu Coreopsideae}

Bidens L., 1753 68/43/0 (Melchert 2010a)

Chrysanthellum Pers., 1807 10/0/0 (Turner 1988)

Coreocarpus Benth., 1844 7/6/0 (Smith 1989)

Coreopsis L., 1753 21/16/0 (Turner 2010c)

Cosmos Cav., 1791 33/27/0 (Castro-Castro et al. 2014)

Dahlia Cav., 1791 39/33/0 (Hansen 2004)

Dicranocarpus A. Gray, 1855 1/0/0 (Turner 2010c)

Electranthera Mesfin, D.J. Crawford \& Pruski, 2015 3/2/0 (Pruski et al. 2015)

Eryngiophyllum Greenm., 1903 2/2/0 (Turner 2010c)

Goldmanella Greenm., 1908 1/0/0 (Turner 2010c)

Henricksonia B.L. Turner, 1977 1/1/0 (Turner 2010c)

Heterosperma Cav., 1794 1/0/0 (Turner 2010c)

Hidalgoa La Llave, 1824 3/2/0 (Turner 2010c)

Thelesperma Less., 1831 9/5/0 (Melchert 2010b)

\section{Tribu Eupatorieae}

Adenostemma J.R. Forst. \& G. Forst., 1776 1/0/0

Ageratella A. Gray ex S. Watson, 1887 1/1/0 (Turner 1995a)

Ageratina Spach, 1841 167/137/0 (Turner 1997)

Ageratum L., 1753 12/5/0 (Cabrera-Rodríguez \& Villaseñor 1987)

Alomia Kunth, 1818 4/4/0 (Cabrera-Rodríguez \& Villaseñor 1987)

Amolinia R.M. King \& H. Rob., 1972 1/0/0

Asanthus R.M. King \& H. Rob., 1972 3/2/0 (Turner 2014)

Bartlettina R.M. King \& H. Rob., 1971 23/14/0 (Turner 2010b)

Brickellia Elliot, 1824 85/57/0 (Turner 1997)

Campuloclinium DC., 1836 1/0/0

Carminatia Moç. ex DC., 1838 4/2/0 (Cabrera-Rodríguez \& Villaseñor 1987)

Carphochaete A. Gray, 1849 7/6/0

Chromolaena DC., 1836 17/9/0

Conoclinium DC., 1836 3/1/0

Critonia P. Browne, 1756 15/5/0

Critoniadelphus R.M. King \& H. Rob., 1971 1/0/0

Decachaeta DC., 1836 8/7/0 (Turner 2011)

Eupatoriastrum Greenm., 1903 7/5/0 (Turner 1994)

Eupatorium L., 1753 1/0/0

Fleischmannia Sch. Bip., 1850 22/6/0

Fleischmanniopsis R.M. King \& H. Rob., 1971 2/0/0

Flyriella R.M. King \& H. Rob., 1972 4/3/0

Gymnocoronis DC., 1836 1/0/0 (Turner 1997)

Hebeclinium DC., 1836 1/0/0

Hofmeisteria Walp., 1846 12/12/0 (Turner 1997)

Isocarpha R. Br., 1817 1/0/0 (Cabrera-Rodríguez \& Villaseñor 1987)

Jaliscoa S. Watson, 1890 3/3/0 (Cabrera-Rodríguez \& Villaseñor 1987)

Koanophyllon Arruda, 1816 23/18/0 (Turner 2009a)

Kyrsteniopsis R.M. King \& H. Rob., 1971 9/7/0

Liatris Gaertn. ex Schreb., 1791 1/0/0 (Turner 1997)

Macvaughiella R.M. King \& H. Rob., 1968 2/1/0 (Robinson 1991)

Malperia S. Watson, 1889 1/0/0 (Turner 1997)

Mexianthus B.L. Rob., 1928 1/1/0 (Turner 1997) 
Apéndice 1.

Continúa.

Microspermum Lag., 1816 8/8/0 (Turner 1997)

Mikania Willd., 1803 18/4/0 (Holmes 1990)

Neomirandea R.M. King \& H. Rob., 1970 2/0/0

Oxylobus (Moc. ex DC.) A. Gray, 1879 7/4/0 (Turner 1997)

Paneroa E.E. Schill., 2008 1/1/0 (Schilling 2008)

Peteravenia R.M. King \& H. Rob., 1971 3/1/0

Piqueria Cav., 1794 6/5/0 (Turner 1997)

Pleurocoronis R.M. King \& H. Rob., 1966 3/2/0 (Turner 1997)

Shinnersia R.M. King \& H. Rob., 1970 1/0/0

Stevia Cav., 1797 116/95/0 (Turner 1997)

Steviopsis R.M. King \& H. Rob., 1971 7/6/0 (Turner 1997)

Tamaulipa R.M. King \& H. Rob., 1971 1/0/0

Trichocoronis A. Gray, 1849 2/1/0 (Turner 1997)

\section{Tribu Gnaphalieae}

Achyrocline (Less.) DC., 1838 5/3/0 (Nesom 1990a)

Anaphalis DC., 1838 1/0/0

Ancistrocarphus A. Gray, 1868 1/0/0

Antennaria Gaertn. 1791 3/0/0

Chionolaena DC., 1836 9/8/0 (Nesom 1990b)

Diaperia Nutt., 1840 1/0/0

Gamochaeta Wedd., 1856 7/0/0 (Freire \& Iharlegui 1997)

Gnaphalium L., 1753 2/0/1

Logfia Cass., 1819 4/0/1

Mexerion G.L. Nesom, 1990 2/2/0 (Nesom 1990d)

Micropus L., 1753 1/0/0

Pseudognaphalium Kirp., 1950 33/1/1

Psilocarphus Nutt., 1841 2/0/0 (Cronquist 1950)

Stylocline Nutt., 1840 3/0/0

*Xerochrysum Tzvelev, 1990 1/0/1 (Villaseñor et al. 2012)

\section{Tribu Gochnatieae}

Nahuatlea V.A. Funk, 2017 7/6/0 (Funk et al. 2017)

\section{Tribu Helenieae}

Amblyolepis DC., 1836 1/0/0

Baileya Harv. \& A. Gray ex A. Gray, 1849 3/0/0 (Turner 1993)

Gaillardia Foug., 1786 12/7/0 (Turner \& Watson 2007)

Helenium L., 1753 11/2/0 (Cabrera-Rodríguez \& Villaseñor 1987)

Hymenoxys Cass., 1828 5/3/0 (Cabrera-Rodríguez \& Villaseñor 1987)

Pelucha S. Watson, 1889 1/1/0

Plateilema (A. Gray) Cockerell, 1904 1/0/0 (Turner 2000)

Psathyrotes (Nutt.) A. Gray, 1853 1/0/0 (Cabrera-Rodríguez \& Villaseñor 1987)

Psilostrophe DC., 1838 4/0/0 (Cabrera-Rodríguez \& Villaseñor 1987)

Tetraneuris Greene, 1898 3/0/0 (Bierner \& Turner 2003)

Trichoptilium A. Gray, 1859 1/0/0

Villanova Lag., 1816 1/1/0

\section{Tribu Heliantheae}

Acmella Rich. ex Pers., 1807 5/2/0 (Cabrera-Rodríguez \& Villaseñor 1987)

Aldama La Llave, 1824 36/32/0

Ambrosia L., 1753 29/12/0 (Cabrera-Rodríguez \& Villaseñor 1987)

Bahiopsis Kellogg, 1863 11/9/0 (Schilling 1990)

Baltimora L., 1771 2/0/0 (Cabrera-Rodríguez \& Villaseñor 1987)

Berlandiera DC., 1836 3/0/0 (Cabrera-Rodríguez \& Villaseñor 1987)

Borrichia Adans., 1763 2/0/0 (Cabrera-Rodríguez \& Villaseñor 1987)

Calanticaria (B.L. Rob. \& Greenm.) E.E. Schill. \& Panero, 2002 5/5/0 (Schilling \& Panero 2002)

Calyptocarpus Less., 1832 2/0/0 (Cabrera-Rodríguez \& Villaseñor 1987)

Chromolepis Benth., 1840 1/1/0

Clibadium L., 1771 1/0/0 (Arriagada 2003)

Damnxanthodium Strother, 1987 1/1/0 (Strother 1987)

Davilanthus E.E. Schill. \& Panero, 2010 7/7/0 (Schilling \& Panero 2010)

Delilia Spreng. 1823 1/0/0 
Apéndice 1.

Continúa.

Dendroviguiera E.E. Schill. \& Panero, 2011 13/13/0 (Schilling \& Panero 2011)

Dicoria Torr. \& A. Gray, 1859 2/1/0

Dugesia A. Gray, 1882 1/1/0

Eclipta L., 1771 1/0/0

Encelia Adans., 1763 13/8/0 (Cabrera-Rodríguez \& Villaseñor 1987)

Engelmannia Torr. \& A. Gray ex Nutt., 1840 1/0/0

Euphrosyne DC., 1836 1/1/0

Flourensia DC., 1836 10/8/0 (Cabrera-Rodríguez \& Villaseñor 1987)

Geraea Torr. \& A. Gray, 1847 2/0/0

Gonzalezia E.E. Schill. \& Panero, 2011 3/3/0 (Schilling \& Panero 2011)

*Guizotia Cass., 1829 1/0/1 (Villaseñor et al. 2012)

Hedosyne Strother, 2000 1/0/0

Helianthella Torr. \& A. Gray, 1842 5/4/0 (Cabrera-Rodríguez \& Villaseñor 1987)

Helianthus L., 1753 10/0/0 (Cabrera-Rodríguez \& Villaseñor 1987)

Heliomeris Nutt., 1848 5/2/0 (Turner 2013a)

Heliopsis Pers., 1807 11/9/0 (Cabrera-Rodríguez \& Villaseñor 1987)

Hybridella Cass., 1821 2/2/0 (Cabrera-Rodríguez \& Villaseñor 1987)

Hymenostephium Benth., 1873 11/7/0 (Turner 2013b)

lostephane Benth., 1873 3/3/0 (Cabrera-Rodríguez \& Villaseñor 1987)

Iva L., 1753 3/1/0 (Cabrera-Rodríguez \& Villaseñor 1987)

Jefea Strother, 1991 4/3/0 (Strother 1991)

Lagascea Cav., 1803 8/5/0 (Cabrera-Rodríguez \& Villaseñor 1987)

Lasianthaea DC., 1836 16/14/0 (Cabrera-Rodríguez \& Villaseñor 1987)

Leuciva Rydb., 1922 1/0/0

Lindheimera A. Gray \& Engelm., 1846 1/0/0

Lundellianthus H. Rob., 1978 4/2/0 (Robinson 1978, Strother 1989)

Melanthera Rohr, 1792 2/0/0 (Cabrera-Rodríguez \& Villaseñor 1987)

Montanoa Cerv., 1825 19/12/0 (Cabrera-Rodríguez \& Villaseñor 1987)

Otopappus Benth., 1873 14/9/0 (Turner 2013c)

Parthenice A. Gray, 1853 1/0/0 (Sauck 1975)

Parthenium L., 1753 11/6/0 (Cabrera-Rodríguez \& Villaseñor 1987)

Perymenium Schrad., 1830 48/43/0 (Cabrera-Rodríguez \& Villaseñor 1987)

Philactis Schrad., 1833 2/1/0 (Cabrera-Rodríguez \& Villaseñor 1987)

Plagiolophus Greenm., 1904 1/1/0 (Villaseñor 1989)

Podachaenium Benth., 1852 4/2/0 (Cabrera-Rodríguez \& Villaseñor 1987)

Ratibida Raf., 1817 6/3/0 (Cabrera-Rodríguez \& Villaseñor 1987)

Rensonia S.F. Blake, 1923 1/0/0 (Strother 1999)

Rojasianthe Standl., \& Steyerm., 1940 1/0/0 (Strother 1999)

Rumfordia DC., 1836 7/5/0 (Cabrera-Rodríguez \& Villaseñor 1987)

Salmea DC., 1813 4/2/0 (Bolick 1991)

Sanvitalia Lam., 1792 5/2/0 (Cabrera-Rodríguez \& Villaseñor 1987)

Sclerocarpus Jacq., 1787 6/4/0 (Villaseñor \& Hinojosa-Espinosa 2011)

Sidneya E.E. Schill. \& Panero, 2011 2/1/0 (Schilling \& Panero 2011)

Simsia Pers., 1807 18/12/0 (Spooner 1990)

Sphagneticola O. Hoffm., 1900 1/0/0 (Strother 1991)

Spilanthes Jacq., 1760 1/0/0 (Cabrera-Rodríguez \& Villaseñor 1987)

Squamopappus R.K. Jansen, N.A. Harriman \& Urbatsch, 1982 1/0/0 (Cabrera-Rodríguez \& Villaseñor 1987)

Synedrella Gaertn., 1791 1/0/0

Tehuana Panero \& Villaseñor, 1996 1/1/0 (Panero \& Villaseñor 1996)

Tithonia Desf., 1789 12/7/0 (Cabrera-Rodríguez \& Villaseñor 1987)

Trichocoryne S.F. Blake, 1924 1/1/0

Trichospira Kunth, 1820 1/0/0

Tuxtla Villaseñor \& Strother, 1989 1/0/0 (Villaseñor \& Strother 1989)

Verbesina L., 1753 164/138/0 (Cabrera-Rodríguez \& Villaseñor 1987)

Vigethia W.A. Weber, 1943 1/1/0 (Weber 1943)

Viguiera Kunth, 1820 9/8/0 (Turner, 2015)

Wamalchitamia Strother, 1991 4/3/0 (Strother 1991)

Wedelia Jacq., 1760 19/17/0 (Strother 1991)

Xanthium L., 1753 3/0/0 (Cabrera-Rodríguez \& Villaseñor 1987)

Zaluzania Pers., 1807 12/11/0 (Turner 2012b)

Zexmenia La Llave, 1824 2/0/0 (Strother 1991)

Zinnia L., 1759 23/17/0 (Cabrera-Rodríguez \& Villaseñor 1987)

Zyzyxia Strother, 1991 1/0/0 (Strother 1991) 
Apéndice 1.

Continúa.

Tribu Inuleae

Blumea DC., 1836 1/0/0

Epaltes Cass., 1818 1/0/0

Pluchea Cass., 1817 9/2/0 (Villaseñor \& Villarreal 2006)

Pterocaulon Elliot, 1823 1/0/0

\section{Tribu Liabeae}

Liabellum Rydb., 1927 5/5/0 (Robinson 1983)

Liabum Adans., 1763 1/0/0

Megaliabum Rydb., 1927 2/1/0 (Robinson 1983)

Sinclairia Hook. \& Arn., 1841 15/7/0 (Turner 1989)

Sinclairiopsis Rydb., 1927 2/2/0 (Funk et al. 2012)

\section{Tribu Madieae}

Achyrachaena Schauer, 1838 1/0/0

Adenothamnus D.D. Keck, 1935 1/1/0

Amblyopappus Hook. \& Arn., 1841 1/0/0

Baeriopsis J.T. Howell, 1942 1/1/0

Calycadenia DC., 1836 1/0/0

Centromadia Greene, 1894 3/1/0

Deinandra Greene, 1897 10/4/0 (Tanowitz 1982)

Eriophyllum Lag., 1816 4/0/0 (Cabrera-Rodríguez \& Villaseñor 1987)

Holocarpha (DC.) Greene, 1897 1/0/0

Hulsea Torr. \& A. Gray, 1858 3/0/0 (Wilken 1975)

Lasthenia Cass., 1834 3/0/0 (Cabrera-Rodríguez \& Villaseñor 1987)

Layia Hook. \& Arn. ex DC., 1838 2/0/0

Madia Molina, 1781 3/0/0

Venegasia DC., 1838 1/0/0 (Turner \& Zippin 1992)

\section{Tribu Millerieae}

Alepidocline S.F. Blake, 1934 5/4/0 (Turner 1990a)

Alloispermum Willd., 1807 10/8/0 (Cabrera-Rodríguez \& Villaseñor 1987)

Aphanactis Wedd., 1856 1/1/0 (Cabrera-Rodríguez \& Villaseñor 1987)

Axiniphyllum Benth., 1872 5/5/0 (González-Zamora \& Villaseñor 2017)

Bebbia Greene, 1885 2/1/0 (Cabrera-Rodríguez \& Villaseñor 1987)

Cymophora B.L. Rob., 1907 4/4/0 (Cabrera-Rodríguez \& Villaseñor 1987)

Desmanthodium Benth., 1872 5/4/0

Dyscritothamnus B.L. Rob., 1922 2/2/0

Faxonia Brandegee, 1894 1/1/0

Galinsoga Ruiz \& Pav., 1794 8/7/0 (Cabrera-Rodríguez \& Villaseñor 1987)

Guardiola Cerv. ex Humb. \& Bonpl., 1807 11/10/0 (Cabrera-Rodríguez \& Villaseñor 1987) Jaegeria Kunth, 1820 8/6/0 (Turner 1984)

Melampodium L., 1753 40/22/0 (Cabrera-Rodríguez \& Villaseñor 1987)

Milleria L., 1753 2/0/0

Oteiza La Llave, 1832 3/3/0

Sabazia Cass., 1827 10/9/0 (Cabrera-Rodríguez \& Villaseñor 1987)

Schistocarpha Less., 1831 8/5/0 (Cabrera-Rodríguez \& Villaseñor 1987)

Selloa Kunth 1820 1/1/0 (Cabrera-Rodríguez \& Villaseñor 1987)

Sigesbeckia L., 1764 3/1/0 (Cabrera-Rodríguez \& Villaseñor 1987)

Smallanthus Mack., 1933 6/3/0 (Cabrera-Rodríguez \& Villaseñor 1987)

Stachycephalum Sch. Bip. ex Benth., 1872 1/1/0

Stenocarpha S.F. Blake, 1915 2/2/0

Tetragonotheca L., 1753 1/0/0 (Turner \& Dawson 1980)

Tridax L., 1753 26/24/0 (Cabrera-Rodríguez \& Villaseñor 1987)

Trigonospermum Less., 1832 6/4/0 (Cabrera-Rodríguez \& Villaseñor 1987)

Zandera D.L. Schulz, 1988 3/3/0

Tribu Mutisieae

Adenocaulon Hook., 1829 1/0/0 (Turner 2009b)

Chaptalia Vent., 1802 10/6/0 (Cabrera-Rodríguez \& Villaseñor 1987)

Leibnitzia Cass., 1822 2/1/0 (Cabrera-Rodríguez \& Villaseñor 1987) 
Apéndice 1.

Continúa.

Tribu Nassauvieae

Acourtia D. Don, 1830 76/67/0 (Cabrera-Rodríguez \& Villaseñor 1987)

Jungia L. f., 1781 2/1/0 (Turner 2009b)

Trixis P. Browne, 1756 19/15/0 (Cabrera-Rodríguez \& Villaseñor 1987)

\section{Tribu Neurolaeneae}

Calea L., 1763 7/3/0 (Cabrera-Rodríguez \& Villaseñor 1987)

Enydra Lour., 1790 1/0/0

Greenmaniella W.M. Sharp, 1935 1/1/0 (Cabrera-Rodríguez \& Villaseñor 1987)

Neurolaena R. Br., 1817 11/8/0 (Cabrera-Rodríguez \& Villaseñor 1987)

Tetrachyron Schltr., 1847 8/7/0 (Cabrera-Rodríguez \& Villaseñor 1987)

\section{Tribu Onoserideae}

Onoseris Willd., 1803 1/0/0 (Cabrera-Rodríguez \& Villaseñor 1987)

\section{Tribu Perityleae}

Amauria Benth., 1844 3/3/0 (Cabrera-Rodríguez \& Villaseñor 1987)

Eutetras A. Gray, 1879 2/2/0 (Cabrera-Rodríguez \& Villaseñor 1987)

Galeana La Llave, 1824 1/0/0

Pericome A. Gray, 1853 2/1/0 (Cabrera-Rodríguez \& Villaseñor 1987)

Perityle Benth., 1844 43/34/0 (Cabrera-Rodríguez \& Villaseñor 1987)

\section{Tribu Senecioneae}

Barkleyanthus H. Rob. \& Brettell, 1974 1/0/0 (Robinson \& Brettell 1974)

Digitacalia Pippen, 1968 4/4/0 (Turner 1990b)

*Emilia Cass., 1817 2/0/2 (Villaseñor et al. 2012)

Erechtites Raf., 1817 2/0/0 (Cabrera-Rodríguez \& Villaseñor 1987)

*Gynura Cass., 1825 1/0/1 (Villaseñor et al. 2012)

*lacobaea Mill., 1754 1/0/1 (Villaseñor et al. 2012)

Lepidospartum (A. Gray) A. Gray, 1883 1/0/0

Nelsonianthus H. Rob. \& Brettell, 1973 2/1/0 (Robinson \& Brettell 1973b)

Packera A. Löve \& D. Löve, 1976 19/13/0 (Freeman \& Barkley 1995)

Pentacalia Cass., 1827 4/2/0 (Robinson \& Cuatrecasas 1978)

Pippenalia McVaugh, 1972 1/1/0 (Cabrera-Rodríguez \& Villaseñor 1987)

Pittocaulon H. Rob. \& Brettell, 1973 5/4/0 (Robinson \& Brettell 1973a)

Psacaliopsis H. Rob. \& Brettell, 1974 4/4/0 (Robinson \& Brettell 1974)

Psacalium Cass., 1826 47/45/0 (Cabrera-Rodríguez \& Villaseñor 1987)

Pseudogynoxys (Greenm.) Cabrera, 1950 2/0/0 (Robinson \& Cuatrecasas 1977)

Robinsonecio T.M. Barkley \& J.P. Janovec, 1996 2/1/0 (Barkley \& Janovec 1996)

Roldana La Llave, 1825 55/43/0 (Robinson \& Brettell 1974, Turner 2005, Funston 2008)

Senecio L., 1753 63/43/2 (Cabrera-Rodríguez \& Villaseñor 1987)

Telanthophora H. Rob. \& Brettell, 1974 6/3/0 (Robinson \& Brettell 1974)

Tetradymia DC., 1838 1/0/0 (Cabrera-Rodríguez \& Villaseñor 1987)

Villasenoria B.L. Clark, 1999 1/1/0 (Clark 1999)

Werneria Kunth, 1818 1/0/0

\section{Tribu Tageteae}

Adenophyllum Pers., 1807 11/8/0 (Strother 1986)

Arnicastrum Greenm., 1903 2/2/0 (Cabrera-Rodríguez \& Villaseñor 1987)

Bajacalia Loockerman, B.L. Turner \& R.K. Jansen, 2003 3/3/0 (Loockerman et al. 2003)

Boeberastrum (A. Gray) Rydb., 1916 2/2/0 (Strother 1986)

Boeberoides (DC.) Strother, 1986 1/1/0 (Strother 1986)

Chrysactinia A. Gray, 1849 6/5/0 (Villaseñor \& Redonda-Martínez, 2009)

Clappia A. Gray, 1859 1/0/0

Comaclinium Scheidw. \& Planch., 1852 1/0/0 (Strother 1986)

Coulterella Vasey \& Rose, 1890 1/1/0

Dyssodia Cav., 1802 4/2/0 (Cabrera-Rodríguez \& Villaseñor 1987)

Flaveria Juss., 1789 16/13/0 (Cabrera-Rodríguez \& Villaseñor 1987)

Gymnolaena (DC.) Rydb., 1915 3/3/0 (Cabrera-Rodríguez \& Villaseñor 1987)

Haploesthes A. Gray, 1849 4/3/0 (Cabrera-Rodríguez \& Villaseñor 1987)

Hydropectis Rydb., 1916 3/3/0 (Turner 1995b)

Jaumea Pers., 1807 1/0/0 
Apéndice 1.

Continúa.

Leucactinia Rydb., 1915 1/1/0

Nicolletia A. Gray, 1845 3/1/0 (Cabrera-Rodríguez \& Villaseñor 1987)

Oxypappus Benth., 1845 1/1/0 (Cabrera-Rodríguez \& Villaseñor 1987)

Pectis L., 1759 44/27/0 (Cabrera-Rodríguez \& Villaseñor 1987)

Porophyllum Adans., 1763 16/11/0 (Cabrera-Rodríguez \& Villaseñor 1987)

Pseudoclappia Rydb., 1923 1/0/0

Sartwellia A. Gray, 1852 3/3/0 (Cabrera-Rodríguez \& Villaseñor 1987)

Strotheria B.L. Turner, 1972 1/1/0 (Cabrera-Rodríguez \& Villaseñor 1987)

Tagetes L., 1753 27/15/1 (Cabrera-Rodríguez \& Villaseñor 1987)

Thymophylla Lag., 1816 14/5/0 (Strother 1986)

Urbinella Greenm., 1903 1/1/0

Varilla A. Gray, 1849 2/1/0 (Turner 1990c)

\section{Tribu Vernonieae}

Bolanosa A. Gray, 1852 1/1/0

*Centratherum Cass., 1817 1/0/1 (Villaseñor et al. 2012)

*Cyanthillium Blume, 1826 1/0/1 (Villaseñor et al. 2012)

Cyrtocymura H. Rob., 1987 1/0/0

Elephantopus L., 1753 1/0/0 (Cabrera-Rodríguez \& Villaseñor 1987)

Eremosis (DC.) Gleason, 1906 21/17/0 (Pruski 2016)

Harleya S.F. Blake, 1932 1/0/0

Leiboldia Schltdl. ex Gleason, 1906 2/2/0 (Jones 1979)

Lepidaploa (Cass.) Cass., 1825 9/3/0 (Pruski 2017)

Lepidonia S.F. Blake, 1936 6/5/0 (Cabrera-Rodríguez \& Villaseñor 1987)

Orthopappus Gleason, 1906 1/0/0

Piptocarpha R. Br., 1816 1/0/0

Pseudelephantopus Rohr, 1792 1/0/0 (Cabrera-Rodríguez \& Villaseñor 1987)

Spiracantha Kunth, 1820 1/0/0

Stenocephalum Sch. Bip., 1863 1/0/0

Stramentopappus H. Rob. \& V.A. Funk, 1987 2/2/0 (Robinson \& Funk 1987)

Struchium P. Browne, 1756 1/0/0

Vernonanthura H. Rob., 1992 8/6/0 (Robinson 1992)

Vernonia Schreb., 1791 14/11/0 (Cabrera-Rodríguez \& Villaseñor 1987) 